\title{
No Sharing! Ein Mixed-Methods-Ansatz zur Analyse von Kurzzeitvermietungen und ihren sozialräumlichen Auswirkungen am Beispiel der Stadt Salzburg
}

\section{No sharing! A mixed methods study of short- term rentals and its socio-spatial implications in the city of Salzburg}

https://doi.org/10.2478/rara-2019-0054

Eingegangen: 27. März 2019; Angenommen: 13. September 2019

Kurzfassung: Kurzzeitvermietungen im Allgemeinen und die Internet-Vermietungsplattform Airbnb im Besonderen stehen gegenwärtig im öffentlichen Fokus. Der Beitrag untersucht am Beispiel der Stadt Salzburg mit einem Mixed-Methods-Ansatz sowohl die räumliche Ausprägung als auch die Akteurstruktur, die sich mit dieser Form des Plattformurbanismus verbinden. Konkret liefert der Beitrag Erkenntnisse hinsichtlich der räumlichen Verteilung, Angebots- und Einnahmenstruktur, Motive der Anbieterinnen/Anbieter und Auswirkungen von Airbnb auf den Wohnungsmarkt. Es wird deutlich, dass die von Airbnb aufgegriffene und beworbene Idee des geteilten Wohnraums im Sinne eines „Homesharing" eine untergeordnete Rolle spielt. Zwischen Hotelgewerbe und Wohnungsvermietung hat sich ein neuer Markt entwickelt, den vor allem kommerzielle Anbieter, die den Airbnb-Markt in Salzburg bestimmen, bedienen. Wir argumentieren, dass mittels Airbnb eine neue Form von rent gap entsteht, der nicht mehr eine vorherige Entwertung von Stadtvierteln vorausgeht. Diese Ertragslücke ergibt sich nun auch in Stadtgebieten, die sowohl in preislicher als auch soziodemographischer Hinsicht als stabil bezeichnet werden können, da innerhalb sehr kurzer Zeit und mittels geringem finanziellem Aufwand Profitsteigerungen möglich sind. Die Auswirkungen auf den Wohnungsmarkt sind vor allem in den touristisch stark frequentierten Innenstadtbereichen Salzburgs erheblich, wodurch Verdrängungsprozesse angestoßen oder intensiviert werden.

Schlüsselwörter: Kurzzeitvermietung, Airbnb, Mixed-Methods-Ansatz, Anbieter, Ertragslücke, Wohnungsmarkt, Salzburg

Abstract: Short-term rentals in general and Airbnb in particular are currently in the public focus. Using the example of the city of Salzburg with a mixed-method approach, this paper examines both the spatial characteristics and the stakeholder structure associated with this form of platform urbanism. Specifically, the paper provides insights into the spatial

\footnotetext{
*Corresponding author: Ass.-Prof. Dr. Christian Smigiel, Universität Salzburg, Fachbereich Geographie und Geologie, Arbeitsgruppe Sozialgeographie, Hellbrunnerstraße 34, 5020 Salzburg, Österreich, E-Mail: christian.smigiel@sbg.ac.at Assoz.-Prof. Dr. Angela Hof, Universität Salzburg, Fachbereich Geographie und Geologie, Arbeitsgruppe Stadt- und Landschaftsökologie, Hellbrunnerstraße 34, 5020 Salzburg, Österreich, ORCID: 0000-0002-0763-110X Karolin Kautzschmann, MSc, Leibniz Universität Hannover, Institut für Wirtschafts- und Kulturgeographie, Schneiderberg 50, 30167 Hannover, Deutschland

Roman Seidl, Technische Universität Wien, Institut für Raumplanung, Operngasse 11, 1040 Wien, Österreich
} 
distribution, supply and revenue structure, motives of hosts and effects of Airbnb on the housing market. It becomes evident that Airbnb's idea of shared housing in the sense of the so-called home sharing plays a subordinate role. A new market has developed between the hotel business and regular apartment rentals, which is determined primarily by commercial providers serving the Airbnb market in Salzburg. We argue that Airbnb creates a new form of rent gap, which is no longer preceded by a prior devaluation of districts. This earnings gap now also arises in urban areas that can be described as stable in terms of both price and socio-demographics, since profit increases can be achieved within a very short period of time and with little financial effort. The effects on the housing market are significant, especially in the heavily touristic frequented inner city areas of Salzburg, which cause or intensify processes of change.

Keywords: Short-term rentals, Airbnb, Mixed-method approach, Hosts, Rent gap, Housing market, Salzburg

\section{Einleitung}

Kurzzeitvermietungen im Allgemeinen und die InternetVermietungsplattform Airbnb im Besonderen werden gegenwärtig intensiv diskutiert. Ablesbar ist dies unter anderem an vielen Berichterstattungen sowohl in lokalen Tageszeitungen (Huber/Fröschl/Veigl 2018; Rossacher 2018) als auch in Leitmedien wie Die Zeit (Twickel 2018), Süddeutsche Zeitung (Kohrs 2019), Der Standard (Putschögl/Zoidl 2018) bzw. anhand investigativer Dokumentationen im Rundfunk. ${ }^{1}$ Auch die jüngsten gerichtlichen Auseinandersetzungen zwischen Kommunen und Airbnb belegen die gesellschaftspolitische Bedeutung dieses Themas. ${ }^{2}$ Airbnb und ähnliche Plattformen werden insbesondere vor dem Hintergrund stark steigender Wohnkosten in vielen Stadtregionen als ,Preistreiber' kritisiert. Weitere (wenngleich weniger stark diskutierte) Bestandteile in der öffentlichen Diskussion sind die Verknappung der lokalen Wohnraumversorgung aufgrund von Kurzzeitvermietungen, die Anpassung lokaler Versorgungsinfrastruktur an Touristen, nicht entrichtete Steuern von Vermieterinnen/Vermietern sowie mögliche Regulationen seitens der öffentlichen Hand.

Auch aufgrund der Novität von Kurzzeitvermietungen und einem komplexen Daten- bzw. Feldzugang gibt es entgegen der intensiven medialen Diskussion bisher nur wenige wissenschaftliche Untersuchungen in den urban studies, die sich mit den oben genannten Themen auseinandersetzen. Dies gilt vor allem für die deutschsprachige Stadtforschung, in der es an Studien fehlt, die konkrete Auswirkungen von Kurzzeitvermietungen via Online-Plattformen untersuchen. Der

1 Dokumentation „Kritisch Reisen: Das System Airbnb - Im Bett mit dem Supervermieter" aus der Reihe „Die Story“, Westdeutscher Rundfunk, Ausstrahlung am 18. Juli 2018; vgl. https://programm. ard.de/TV/wdrfernsehen/kritisch-reisen--das-system-airbnb-/ eid_28111754896448 (14.08.2019).

2 Bayrisches Verwaltungsgericht München, Urteil vom 12. Dezember 2018, Aktenzeichen: M 9 K 18.4553. vorliegende Beitrag greift dieses Forschungsdefizit auf und liefert beispielhaft für den Kontext der Stadt Salzburg eine kritische Bestandsaufnahme und Analyse. Konkret verfolgen wir zwei Anliegen. Erstens geht es um eine empirisch fundierte Analyse der Akteurstruktur. Darauf aufbauend sollen Einschätzungen hinsichtlich der Auswirkungen auf den Wohnungsmarkt getroffen werden. Hierzu kombiniert er quantitative und qualitative Methoden der empirischen Sozialforschung mit einem informationstechnischen Ansatz, um räumliche, ökonomische und soziale Dimensionen von Kurzzeitvermietungen (über Airbnb) zu analysieren. Drei Fragestellungen werden dezidiert betrachtet: i) Welche Akteur- und Anbieterstruktur liegt in der Stadt Salzburg im Kontext von Airbnb vor? ii) Welche Motivlagen kennzeichnen die Anbieterstruktur von Airbnb in der Stadt Salzburg? iii) Inwiefern beeinflussen Kurzzeitvermietungen den Salzburger Wohnungsmarkt? Während die ersten beiden Fragen auf die aktuelle Akteurebene abzielen, greift die dritte Frage diese Erkenntnisse auf und blickt auf die mittel- und langfristigen strukturellen Auswirkungen dieser Form des Plattformurbanismus.

Im Anschluss an diese Einleitung wird im folgenden Kapitel zunächst der bisherige Forschungsstand zu Kurzzeitvermietungen zusammengefasst, es werden Forschungslücken geortet und es wird zudem ausgeführt, welche konzeptionellen Argumente der internationalen Stadtforschung im vorliegenden Beitrag diskutiert werden. In Kapitel 3 sind die wohnungs- und stadtpolitischen Rahmenbedingungen - inclusive der von uns eingebetteten Kurzzeitvermietungen - in der Stadt Salzburg skizziert. Nach einer ausführlichen Darstellung des methodischen Vorgehens in Kapitel 4 diskutiert Kapitel 5 die wesentlichen Erkenntnisse hinsichtlich der oben genannten Fragestellungen. Wir beschließen diesen Beitrag mit einem kurzen Fazit, das unter anderem einen Ausblick auf zukünftige Forschungsthemen im Bereich Kurzzeitvermietungen gibt. 


\section{Forschungsstand}

Forschungsarbeiten, die sich mit dem starken Anstieg von Kurzzeitvermietungen in Stadtregionen auseinandersetzen, gibt es bisher in der deutschsprachigen Stadt- und Regionalforschung nur wenige. Diese wenigen vorliegenden Studien betrachten Airbnb und ähnliche Plattformen entweder aus der Perspektive der Tourismusforschung oder sie fokussieren auf methodische Aspekte und diskutieren hierbei insbesondere quantitative Ausprägungen. $\mathrm{Zu}$ nennen sind in diesem Zusammenhang die Arbeiten von Kagermeier, Köller und Stors (2015) sowie Stors und Kagermeier (2017), die Airbnb als Teil des New Urban Tourism sehen und die diversen Sichtweisen von Anbieterinnen und Anbietern, Bewohnerinnen und Bewohnern in den Kontext hochgradig individualisierter und flexibilisierter Gesellschaftsverhältnisse einordnen (Stors/Kagermeier 2017: 219). Hörz (2016) sowie Skowronnek, Vogel und Parnow (2015) waren Vorreiter, was die graphische und raumbezogene Handhab- und Sichtbarmachung von online (auf der Airbnb-Plattform) verfügbaren Daten anbetrifft (Hörz 2016: 36). Den genannten Studien ist gemein, dass sie sich allesamt mit Kurzzeitvermietungen in Berlin beschäftigen. Weitere wissenschaftliche Untersuchungen liegen darüber hinaus nur für Wien vor, wo mittels eines quantitativen Zugangs erste Aussagen über die wohnungsmarktspezifischen Auswirkungen von Airbnb gemacht werden können (Seidl/Plank/Kadi 2017). ${ }^{3}$

Ähnlich wie bereits für den deutschsprachigen Kontext skizziert, wurden Kurzzeitvermietungen international zunächst in den Tourism Studies thematisiert. Insbesondere die Debatte um overtourism hat dazu geführt, dass auch Kurzzeitvermietungen verstärkt in den Blick rück(t)en. In diesem Kontext werden sie als Teil stetig wachsender Touristenströme gesehen, was zunehmend Widerstände und Proteste der ortsansässigen Bevölkerung auslöst. Insbesondere in Städten wie Barcelona, Berlin, Dubrovnik, Palma de Mallorca oder Venedig gibt es wachsenden Protest gegen die Touristifizierung von ganzen Stadtgebieten (Novy/Colomb 2017: 3). Dabei ist overtourism prinzipiell kein neues Phänomen. Allerdings tritt er nun auch abseits der klassischen Touristenpfade und Hotspots auf und sorgt für weitreichende Transformationen des Städtischen (Novy/Colomb 2017; Frisch/ Sommer/Stoltenberg et al. 2019).

In der internationalen Stadtforschung wird in jüngster Zeit vermehrt diskutiert, welche Auswirkungen Kurzzeitvermietungen auf lokale Wohnungsmärkte haben.

3 Näheres zu den methodischen Überschneidungen in Kapitel 4.
Angetrieben unter anderem durch kritische (Daten-) Aktivisten wie Murray Cox und Tom Slee wurden in den letzten Jahren vor allem Airbnb-Hotspots wie Barcelona, London, New York, Palma de Mallorca und Paris untersucht. ${ }^{4}$ Konzeptionell schlagen vor allem die Arbeiten von Cócola Gant (2016, 2018), Cócola Gant und Gago (2019), Roelofsen (2018), Wachsmuth und Weisler (2018) sowie Yrigoy (2018) eine Brücke zur kritischen Stadtforschung und der damit verbundenen kritischen Auseinandersetzung mit den sozialräumlichen und politökonomischen Implikationen von Kurzzeitvermietungen. Insbesondere die Konzentration auf bestimmte Stadtviertel (Cócola Gant 2016: 5; Roelofsen 2018: 324), direkte und indirekte Verdrängungsprozesse von Bewohnern und Infrastruktur (Cócola Gant 2018: 289) sowie die Schaffung einer neuen Ertragslücke (rent gap) (Wachsmuth/Weisler 2018: 1153; Yrigoy 2018) sind wesentliche Erkenntnisse dieser Studien. ${ }^{5}$ Letztgenannter Punkt ist in mittel- und langfristiger Hinsicht von Bedeutung, verdeutlicht er doch, dass mit Kurzzeitvermietungen eine neue ökonomische Verwertungslogik von Wohnraum verbunden ist, die vor allem in Städten mit starkem Städtetourismus (teilweise bereits jetzt) zu Verwerfungen führt. Wachsmuth und Weisler (2018: 1154) zeigen, dass Airbnb und ähnliche Plattformen zu außerordentlichen Profitsteigerungen auf Seiten der Vermieterinnen/Vermieter führen, die in sehr kurzen Zeiträumen auftreten oder erwartet werden. Des Weiteren sind diese Profitsteigerungen ohne große Investitionen möglich, was die lokalen Wohnungsmärkte und vor allem einkommensschwächere Haushalte unter Druck setzt. Yrigoy (2018: 15) belegt am Beispiel von Palma de Mallorca, dass Kurzzeitvermietungen für die Gentrifizierungsforschung insofern ein Novum darstellen, da auch Mieteinnahmen nun eine attraktive potenzielle Grundrente darstellen, die einerseits zu Verdrängung von Langzeitbewohnerinnen und -bewohnern und andererseits zum Anstieg der Wohnkosten führt.

Der vorliegende Beitrag geht in konzeptioneller Hinsicht insbesondere den letztgenannten Zusammenhängen für den Salzburger Kontext nach und diskutiert, welche ökonomischen Verwertungspraktiken und Logiken sich beobachten lassen. Hierbei stützt er sich in

4 Vgl. https://insideairbnb.com (14.08.2019).

5 Arbeiten, die sich mit der Reaktion der Gemeinden bzw. der Stadtplanung beschäftigen, gibt es bisher mit Ausnahme der Londoner Studie von Ferreri und Sanyal (2018), die Herausforderungen und Widersprüchlichkeiten von Urban Governance im Zusammenhang mit Kurzzeitvermietungen analysieren, kaum. Dies ist eine der zahlreichen Forschungslücken, denen sich die Stadt- und Regionalforschung in nächster Zeit widmen sollte. 
empirischer Hinsicht auf eine umfangreiche quantitative und qualitative Untersuchung von Kurzzeitvermietungen und erweitert somit die bisherige Forschung. Konkret untersuchen wir sowohl die Anzahl, Lage, Struktur und Anbieter der Airbnb-Angebote als auch mittels qualitativer Interviews mit Anbieterinnen/Anbietern die Motivlagen und den sozioökonomischen Hintergrund dieser Akteure. Diese Erkenntnisse werden vor dem Hintergrund der Wohnungsfrage in Salzburg eingeordnet.

\section{Kurzzeitvermietungen und die Wohnungsfrage in der Stadt Salzburg - der Forschungskontext}

Die Stadt Salzburg ist eine seit Jahrzehnten auf den Tourismus ausgerichtete Stadt. Insbesondere seit Mitte der 2000er Jahre sind die Ankunfts- und Nächtigungszahlen nochmals um bis zu 60-80 \% gestiegen (Stadt Salzburg 2019: 11 ff.). ${ }^{6}$ Bei einer Einwohnerzahl von knapp 156.000 verbucht die ,Mozartstadt' gegenwärtig mehr als 3 Millionen Nächtigungen sowie 9 Millionen Tagestouristen pro Jahr (Neuhold 2018: 16; Stadt Salzburg 2019: 11). Selbst von der Tourismusindustrie finanzierte Studien attestieren Salzburg mittlerweile, dass die Stadt sich in der "Falle des Massentourismus" (Roland Berger Consulting 2018: 8) befindet. Dies verdeutlicht auch eine weitere Kennziffer. Salzburg liegt mit 20 Übernachtungen je Einwohner im europaweiten Vergleich mit ähnlich großen touristischen Destinationen, die mehr als eine Million Übernachtungen aufweisen, auf Rang 1 in punkto Tourismusintensität (Roland Berger Consulting 2015: 17). Besonders anschaulich ist dies in der Salzburger Altstadt zu beobachten, wo unter anderem aufgrund der starken Touristifizierung die Einwohnerzahlen zwischen 2008 und 2015 um $15 \%$ sanken, während sie stadtweit im gleichen Zeitraum um $3 \%$ gestiegen sind (Stadt Salzburg 2013, 2018; eigene Berechnungen).

Zudem ist Salzburg eine der teuersten Städte Österreichs, was die Wohnkosten anbetrifft. Dies gilt sowohl für Baulandpreise als auch für Mieten oder Kaufpreise von Wohnungen und Häusern (Zeller/Van-Hametner/ Smigiel et al. 2018: 605). Die Ursachen sind vielfältig und

$6 \quad$ Vgl. auch https://www.stadt-salzburg.at/internet/bildung kultur/salzburg_in_zahlen/tourismusstatistik_u_333039/ tourismusstatistik_uebernachtungen_1991_356025.htm (14.08.2019). nicht nur auf der lokalen Ebene oder im Tourismus zu suchen. Sie reichen von einer zunehmenden Finanzialisierung im Bereich Wohnen, die sich auch in Salzburg in der stark steigenden Nachfrage nach Anlage- und Vorsorgewohnungen äußert, veränderten institutionellen Rahmenbedingungen auf der nationalen Ebene (Rückgang der Förderung von öffentlichem Wohnungsbau und Deregulierungen im Bereich des Mietrechts) bis zu einer schwach ausgeprägten Regulation im Bereich Wohnen auf regionaler Ebene (Van-Hametner/Smigiel/Kautzschmann et al. 2019: 239 f.).

Gleichwohl gibt es auch tradierte, lokale Ursachen, die die Wohnungsfrage wesentlich prägen. Anders als beispielsweise in Wien beträgt der kommunale Wohnungsbestand in Salzburg weniger als 3 \% (Stadt Salzburg 2018: 18). Dominiert wird der Wohnungsmarkt von privaten Eigentümern, die mehr als $65 \%$ des Wohnungsbestandes besitzen. Ähnliches gilt auch für die Struktur von verfügbarem Bauland. Hinzu kommt aber auch eine restriktive Raumplanung, die große Bereiche unter den Schutz einer dauerhaften Grünlanddeklaration gestellt hat, wodurch eine proaktive Wohnungs- und Stadtpolitik wesentlich erschwert wird (Dopsch/Hoffmann 2008: 612).

\section{Methodisches Vorgehen}

Dieser Beitrag basiert auf einer quantitativen und qualitativen Untersuchung des Airbnb-Angebots in der Stadt Salzburg. Hierzu wurden mittels einer speziell entwickelten Methode, die bereits für Wien angewandt wurde (Seidl/Plank/Kadi 2017), alle Airbnb-Angebote für zwei Stichtage im Juni 2017 und Juni 2018 erhoben und ausgewertet. ${ }^{7}$ Nach einer Bestimmung der Anbieterstruktur wurden leitfadengestützte Tiefeninterviews geführt. Die von uns interviewten Airbnb-Anbieter repräsentieren mehr als $10 \%$ der gesamten Salzburger Airbnb-Angebote, wodurch konkrete Aussagen über räumliche Verteilung, Angebotsstruktur, Motive der Anbieterinnen/ Anbieter und Auswirkungen auf den Wohnungsmarkt getroffen werden können.

7 Weiterführende methodische Details zu Berechnungen und Schätzungen finden sich auf der Website https://wherebnb.in/wien/ (15.08.2019). Die in der Wiener Studie angewandte quantitative Erhebungsmethode haben wir in unserer Salzburger Untersuchung reproduziert. 


\subsection{Quantitativer Zugang mittels Web Scraping und räumlicher Analyse}

\subsubsection{Datenerhebung mittels Web Scraping und Validierung der Daten}

Die Erhebung der Daten erfolgt mittels Web Scraping. Web Scraping steht für Methoden, die Daten von Webseiten automatisiert auslesen und systematisch erheben, um sie für eine Analyse oder Anwendung aufzubereiten. In unserer Untersuchung wurden automatisiert Zugriffe auf den Webservice der Airbnb-Plattform ausgeführt. Diese Zugriffe entsprechen der Suche nach einem Angebot für eine Unterkunft auf der Webseite. Damit wird mittels einer Suchstrategie systematisch nach allen zu spezifischen Stichtagen verfügbaren Angeboten gesucht.

Für diese Angebote werden dann die entsprechenden Detailanfragen für die Ermittlung der Preise, der Buchungskalender, der Bewertungen und Details wie etwa Kapazität, Anbieterin/Anbieter oder Beschreibung mittels weiterer Anfragen an den Webservice erhoben. Die Detaildaten zu den einzelnen Angeboten werden in einer Datenbank gespeichert. Die Konsistenz der Daten wird mittels mehrerer Verfahren geprüft. Einerseits garantiert eine relationale Datenbank die Typsicherheit in den entsprechenden Feldern, andererseits stellt sie die Relationen wie etwa zwischen Bewertungen und Angeboten sicher.

\subsubsection{Vergleich Web-Scraping mit anderen Datensätzen und Plausibilitätsprüfung}

Die mit der oben beschriebenen Web-Scraping-Technik für Testzwecke erhobenen Daten wurden mit Daten aus anderen Quellen (AirDNA, Insideairbnb) ${ }^{8}$ verglichen. In den wesentlichen Dimensionen sind die Ergebnisse stets vergleichbar. Ein Unterscheid liegt darin, dass die Stichtagerhebung im Vergleich zu anderen Quellen tendenziell weniger Ergebnisse liefert als Erhebungsmethoden, die Angebote über einen gewissen Zeitraum betrachten und mit einbeziehen. Hier lässt sich aber argumentieren, dass die Stichtagerhebung für beliebige Zeitpunkte, die in unserer Studie außerhalb saisonaler Höhepunkte liegt, repräsentativer ist. Wenn inaktive Angebote gefiltert werden, ergeben sich so deutlich geringere Abweichungen.

8 https://www.airdna.co (15.08.2019); http://insideairbnb.com/ behind.html (15.08.2019).
Des Weiteren wurde mittels vorangegangener Testerhebungen die Stabilität der Ergebnisse über die Zeit grob geprüft. Es konnte zumindest für einen beschränkten Zeitraum eine in der Struktur relativ stabile Angebotslandschaft festgestellt werden. Schließlich lässt sich die Plausibilität der Ergebnisse auch durch den Vergleich der Verteilung der Angebote über die Stadt mit der räumlichen Struktur der Stadt bezüglich Wohnen und Tourismus überprüfen. Hier und in allen anderen Dimensionen ergaben sich keinerlei auffällige Abweichungen, die eine weitergehende und tiefere Fehlersuche erfordert hätten.

\subsubsection{Ortsunschärfe und räumliche Zuordnung}

Legt man um die auf der Airbnb-Plattform gefundenen Angebote in Salzburg jeweils einen Kreis mit einem 500-m-Radius (in seltenen Fällen 250 m), erkennt man eine Ungleichverteilung der Angebote auf die Stadt. Sie können den administrativen Grenzen der Stadt (Zählbezirke) nicht flächen- oder objektscharf zugeordnet werden. Daher haben wir um jeden auf der Webseite angegebenen Lagepunkt einen Kreis mit einem Radius von $500 \mathrm{~m}$ gelegt. Die Lageanteile dieses Kreises in den Zählbezirken ergeben einen Anteil, in dem dieses Angebot zu den Ergebnissen für die Stadt oder den Zählbezirk beiträgt. Mit dieser Methode werden kleinere Zählbezirke, welche höhere Dichten als die Nachbarbezirke aufweisen, unterschätzt und jene überschätzt, bei denen die Dichten eher fallend sind. Daher sind die berechneten Konzentrationen etwa in der kleinteiligen Salzburger Altstadt wohl tendenziell niedriger als jene, die wirklich vorliegen (vgl. Abbildung 1).

\subsubsection{Aktive Angebote, Schätzung der Auslastung und Einnahmen}

Aktive Angebote sind von allen gefundenen Angeboten all jene Angebote, die entweder im letzten Jahr eine Bewertung hatten oder im nächsten Jahr zur Buchung angeboten werden. Buchungen lösen oft Bewertungen aus. Ein hoher Anteil an bewerteten Buchungen wird von Seiten der Plattform Airbnb gewünscht, das zeigt sich etwa daran, dass für das Prädikat „Superhost" eine hohe Bewertungsrate gefordert wird.

Aus diesen Bewertungen lässt sich eine Auslastung der Unterkünfte schätzen. Dafür benötigt man zwei Annahmen: den Anteil der Buchungen, die zu Bewertungen führen (die Bewertungsrate) und die Aufenthaltsdauer. Zur Bewertungsrate existieren zwei bekannte 
Quellen. Eine Aussage, die Brian Chesky, Geschäftsführer von Airbnb, zugeschrieben wird, spricht von $72 \%$, eine Auswertung einer gerichtlich erzwungenen Datenherausgabe für New York kommt auf $30,5 \%{ }^{10}$ Für eine vorsichtige Schätzung der Bewertungsrate wurde eine Rate von $50 \%$ angenommen. ${ }^{11}$ Damit wird aus der Anzahl der Bewertungen im letzten Jahr (oder seit der ersten Bewertung, wenn das Angebot erst kürzer besteht) eine Anzahl an Buchungen pro Jahr errechnet. ${ }^{12}$

Für die mittlere Aufenthaltsdauer im Bereich von Ferienwohnungen gibt es bisher keine veröffentlichten Daten für die Stadt Salzburg. Es gibt lediglich Statistiken zur Aufenthaltsdauer in Hotels, die sich jedoch wesentlich von der Bleibedauer in Ferienwohnungen unterscheiden (vgl. Stadt Salzburg 2019). In unserer Untersuchung gehen wir von einer mittleren Aufenthaltsdauer von vier Tagen aus. Das entspricht dem kürzesten von Airbnb für eine vergleichbare Stadt veröffentlichten Mittelwert. ${ }^{13}$ Eine aktuelle Airbnb-Auftragsforschung kommt auf der Basis von Befragungen in Österreich, deren Datengrundlage jedoch nicht transparent gemacht wird, zu einem Wert von 3,7 Tagen für das Bundesland Salzburg (EcoAustria 2019: 12).

Kombiniert man die geschätzte Auslastung schließlich mit Preisen und zieht entsprechende Gebühren ab, so lassen sich die Bruttoeinnahmen schätzen, die die Anbieter durch Kurzzeitvermietungen ihrer Unterkünfte erzielen. Dafür braucht man noch Preisangaben zu den Angeboten und Annahmen zu den Gebühren. Die zum jeweiligen Stichtag erhobenen Preise können nicht zwingend als immer gültig angesehen werden. Zunehmend werden Preise nach Auslastung flexibel gesetzt. Airbnb empfiehlt Anbietern die Verwendung eines Preismodells, bei dem ein von der Airbnb-Plattform gesteu-

9 https://www.quora.com/What-percent-of-Airbnb-hosts-leavereviews-for-their-guests (15.08.2019).

10 https://sfbos.org/sites/default/files/FileCenter/Documents/52601BLA.ShortTermRentals.051315.pdf (15.08.2019).

11 Eine vergleichbare Methode findet sich auch bei Seidl/Plank/ Kadi (2017) und http://insideairbnb.com/behind.html (15.08.2019).

12 Bis dato gibt es, nach unserem Kenntnisstand, keine anderen wissenschaftlichen Quellen. Es muss zudem angemerkt werden, dass mit zunehmender Professionalisierung der Anbieterstruktur die Bewertungsrate tendenziell steigt.

$13 \mathrm{http}: / /$ blog.atairbnb.com/economic-impact-airbnb/ (15.08.2019). Auf dieser Basis lässt sich dann mittels Multiplikation der Bewertungen pro Jahr und der mittleren Aufenthaltsdauer eine Anzahl an ausgelasteten Tagen und damit auch eine Auslastungsrate schätzen. Diese modellierte Rate liegt oft unrealistisch hoch. Um hier eine eher vorsichtige Schätzung zu bewahren, wurde die Auslastungsrate (wie auch bei Seidl/Plank/ Kadi 2017) auf $70 \%$ begrenzt. erter Automat innerhalb eines Fensters die Preise selbstständig steuert. Es handelt sich demnach bei den erhobenen Preisen eher um Preise für den günstigsten verfügbaren Tag. Somit werden die Preise wohl meist eher unterschätzt. Etwaige Nebengebühren, die sehr häufig für Reinigung und in seltenen Fällen für zusätzliche Personen anfallen, werden von uns auf die mittlere Nächtigungsdauer aufgeteilt und mit einberechnet. Von diesem Basispreis werden dann noch die Vermietergebühren inklusive Umsatzsteuer (3,6 \%) abgezogen. Die gesamten Bruttoeinnahmen ergeben sich somit, indem man den Nettopreis mit der geschätzten (und begrenzten) Belegung multipliziert.

\subsubsection{Dauerhaft dem Wohnungsmarkt entzogene Wohnungen}

Ein zunehmender Kritikpunkt an Kurzzeitvermietungen ist, dass sie lokalen Wohnungsmärkten dauerhaft Wohnungen entziehen. Um dies empirisch zu untersuchen, haben wir in quantitativer Hinsicht Schwellenwerte festgelegt, nach denen eine Wohnung dem regulären Wohnungsmarkt nicht mehr zur Verfügung steht. Analysen zu den Auswirkungen von Airbnb auf den Wohnungsmarkt arbeiten in der Regel mit einem Auslastungsgrad, über dem eine Vermietung an Langzeitbewohnerinnen und -bewohner unwahrscheinlich ist. Wachsmuth und Weisler (2018: 1164) definieren zum Beispiel einen Schwellenwert von 60 Tagen pro Jahr für häufig vermietete Wohnungen. ${ }^{14}$ Das entspricht einer Vermietung von etwas mehr als einem Tag pro Woche. Wir verwenden ebenso eine Auslastung von 60 Tagen pro Jahr und definieren, in Anlehnung an Wachsmuth und Weisler (2018), zusätzlich noch eine notwendige Verfügbarkeitsdauer von 120 Tagen pro Jahr. Damit filtern wir zusätzlich jene Angebote heraus, die zwar länger als 60 Tage vermietet wurden, aber trotzdem nur zeitweise, etwa nur jedes Wochenende verfügbar sind. Wohnungen, die mehr als 60 Tage im Jahr belegt sind (hohe Auslastung) und mehr als 120 Tage im Jahr zur Buchung verfügbar sind (hohe Verfügbarkeit) definieren wir als dem Wohnungsmarkt entzogene Wohnungen.

14 Die gleiche Dauer wird auch auf http://insideairbnb.com/ behind.html (15.08.2019) angenommen. 


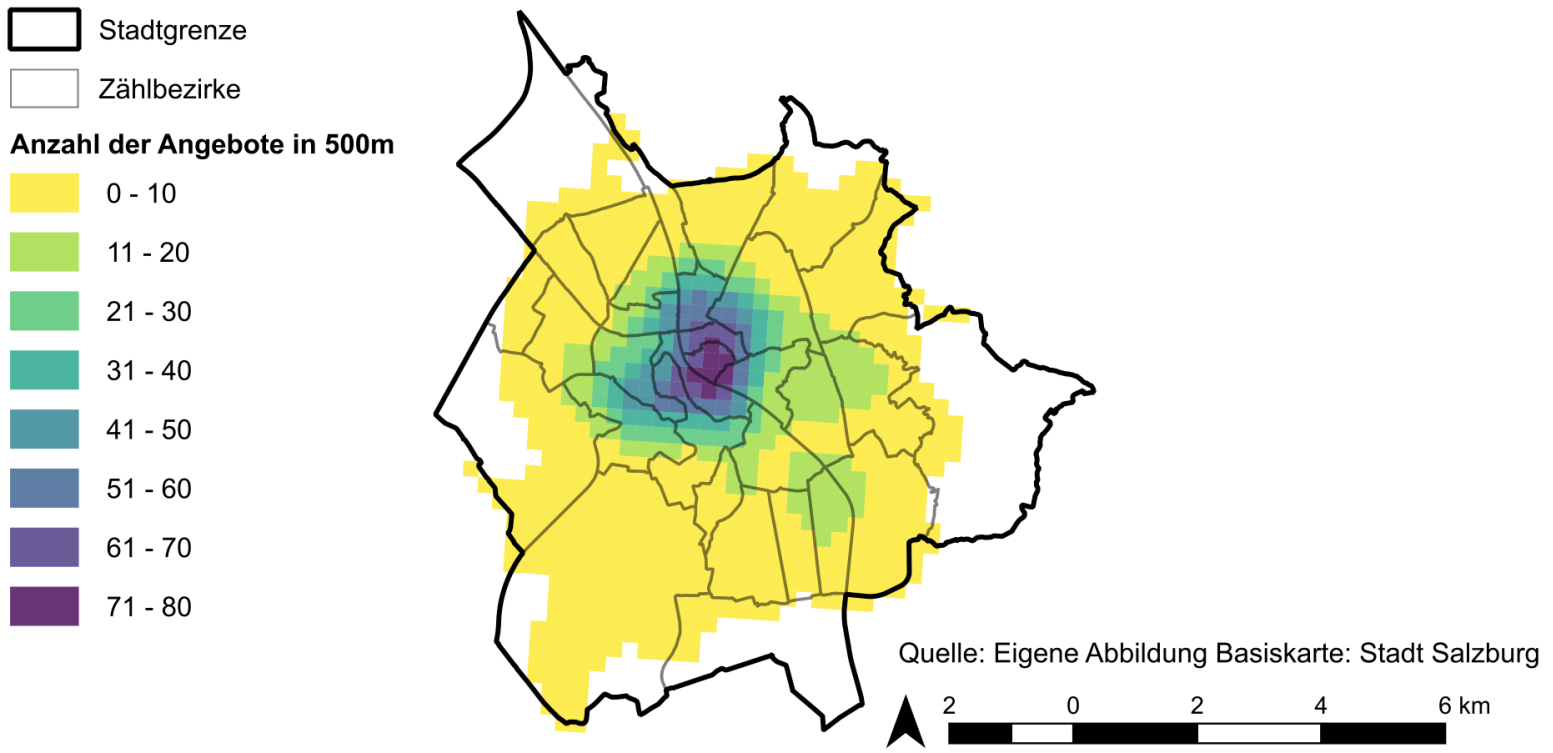

Abbildung 1: Räumliche Verteilung und Dichte der Airbnb-Angebote in der Stadt Salzburg

\subsection{Qualitative Analyse der Anbieterstruktur}

\subsubsection{Lageindikator und Anbietertypologie}

Mit der Methode Web Scraping erhält man einen Überblick hinsichtlich der räumlichen Verteilung von Kurzzeitvermietungen. Konkret zeigt sich für den Salzburger Kontext, dass vor allem in den Salzburger Altstadtvierteln eine hohe Konzentration von Airbnb-Unterkünften vorhanden ist (vgl. Abbildung 1). Anhand der räumlichen Verteilung lassen sich vier Abstufungen (vgl. Abbildung 1 , Farben violett, blau, grün und gelb) erkennen, in die die 32 Salzburger Stadtteile (Zählbezirke) fallen. Diese räumliche Verteilung ist zugleich ein Indikator für den Kommerzialisierungs- bzw. Professionalisierungsgrad von Airbnb in den Zählbezirken. ${ }^{15}$ Wir gehen davon aus, dass dort, wo die höchsten Einnahmen generiert und anteilsmäßig viele Wohnungen angeboten werden, Kurzzeitvermietungen und deren Verwaltung der Wohnungen entsprechend professionell betrieben werden.

Auf der Basis dieser Abstufung und anhand der vorhandenen Detailangaben haben wir im Folgenden

15 Den Professionalisierungsgrad haben wir im qualitativen Teil der Untersuchung nach den folgenden Kriterien bestimmt: Servicepersonal, Gebrauch von Verwaltungssoftwares, Aktivität auf anderen Plattformen, persönlicher und privater Kontakt zu Gästen, eigene Homepage, Outsourcing von Arbeitsschritten. Hinzu kommt der Kommerzialisierungsgrad, den wir wie folgt definiert haben: Anzahl und Grad der Airbnb-Nutzung. eine Typisierung vorgenommen, die in der qualitativen Untersuchung (leitfadengestützte Interviews) getestet und verfeinert wurde. Die Zusammenführung der unterschiedlichen Erkenntnisse zeigt Tabelle 1. Die Anbieter in der Stadt Salzburg lassen sich in zwei Gruppen und vier Kategorien unterteilen (vgl. Tabelle 1).

In einem ersten Schritt unterscheiden wir bezüglich der Anzahl der Angebote. Anbieterinnen/Anbieter der Gruppe A haben nicht mehr als ein Airbnb-Angebot. Anbieterinnen/Anbieter der Gruppe B haben mindestens zwei oder mehr Angebote. Dies impliziert bei Gruppe B eine kommerzielle Nutzung von Airbnb, weil niemand mit zwei Wohnungen in derselben Stadt als Hauptwohnsitz gemeldet sein kann.

Gruppe A wiederum unterteilt sich in die Anbieterkategorien 1 und 2, wobei Anbieterinnen und Anbieter der Kategorie 1 nur ein geteiltes Zimmer oder ihre Wohnung als Zwischennutzung zur Verfügung stellen. Diese Eigenschaften lassen uns davon ausgehen, dass es sich hierbei um eine Form von Homesharing handelt. Anbieterinnen/Anbieter der Kategorie 2 hingegen bieten eine Wohnung oder ein Zimmer teils temporär, teils dauerhaft für Airbnb-Gäste an und bewegen sich damit in einem Graubereich zwischen Homesharing und kommerzieller Nutzung, da hiermit bereits ein regelmäßiges Einkommen generiert wird.

Gruppe B wird unterteilt in die Anbieterkategorien 3 und 4 , weil beide mindestens zwei oder mehr Wohnungen bzw. Zimmer auf Airbnb zur Verfügung stellen. In beiden Fällen handelt es sich um kommerzielle Anbie- 
Tabelle 1: Typen von Airbnb-Anbietern in Salzburg

\begin{tabular}{|c|c|c|c|}
\hline \multicolumn{2}{|l|}{ Gruppe A (1 Angebot) } & \multicolumn{2}{|c|}{ Gruppe B (mindestens 2 Angebote) } \\
\hline Kategorie 1 & Kategorie 2 & Kategorie 3 & Kategorie 4 \\
\hline Ein Angebot (geteiltes & Ein Angebot (Wohnung oder & 2-5 Angebote zur dauerhaften & Mehr als fünf Angebote zur dauerhaften \\
\hline $\begin{array}{l}\text { Zimmer oder geteilte } \\
\text { Wohnung) }\end{array}$ & $\begin{array}{l}\text { Haus nur für Kurzzeitvermie- } \\
\text { tung) }\end{array}$ & $\begin{array}{l}\text { Kurzzeitvermietung; Service- } \\
\text { personal (nur für Reinigung) }\end{array}$ & $\begin{array}{l}\text { Kurzzeitvermietung; Servicepersonal, } \\
\text { Outsourcing von Dienstleistungen }\end{array}$ \\
\hline Nicht dauerhaft & $\begin{array}{l}\text { Nicht dauerhaftes oder dauer- } \\
\text { haftes Angebot }\end{array}$ & Dauerhafte Angebote & Dauerhafte Angebote \\
\hline Homesharer & $\begin{array}{l}\text { Zwischen Homesharing und } \\
\text { kommerzieller Nutzung }\end{array}$ & Kommerzielle Anbieter & Professionelle / Kommerzielle Anbieter \\
\hline
\end{tabular}

ter. Jedoch wird die Verwaltung der Airbnb-Angebote der Kategorie 3 noch von den Anbieterinnen oder Anbietern selbst organisiert. Anders ist es bei Kategorie 4, den sogenannten Top-Anbietern (mehr als fünf Angebote). Hier werden Wohnungen auf Airbnb und anderen Plattformen in großem Stil vermietet. Die Verwaltung wird nicht mehr von den Anbietern selbst organisiert, sondern ist ausgelagert und wird von spezialisierten Dienstleistern ausgeführt. Die Kurzzeitvermietung von Wohnungen/Zimmern ist Haupteinnahmequelle und wird hauptberuflich ausgeübt. Nur in Einzelfällen handelt es sich hierbei um gewerbliche Betriebe, die über Airbnb Hotelzimmer anbieten.

\subsubsection{Leitfadengestützte Interviews}

Zur Bestimmung der Interviewpartner ${ }^{16}$ wurde im folgenden Schritt eine geschichtete Stichprobe gezogen. Hierzu wurde allen gelisteten Angeboten eine Zufallszahl zugeteilt. Konkret wurden aus den vier Zählbezirksgruppen (violett, blau, grün, gelb) 49 Zahlen (Angebote) gezogen. Nach Bereinigung von Doppelnennungen (von Anbietern mit mehreren Angeboten und nicht mehr aktuellen Links) wurden 44 Anbieter kontaktiert, wodurch 13 qualitative Interviews mit Personen zustande kamen. Die Länge der Interviews lag zwischen 25 und 80 Minuten. Insgesamt stehen diese 13 Anbieter für 73-80 AirbnbAngebote (vgl. Tabelle 2), womit sie letztendlich mehr als $10 \%$ aller Airbnb-Angebote in der Stadt Salzburg abdecken. Aufgrund des vorab skizzierten methodischen Zugangs (räumliche Verortung der Airbnb-Angebote, detaillierte und vergleichbare Informationen zu allen

16 Im Sinne der Lesbarkeit des Textes wird in den folgenden Kapiteln die generische Substantivform, die alle Geschlechter einschließt, verwendet.
Tabelle 2: Anzahl der Airbnb-Angebote pro Interviewpartnerin bzw. Interviewpartner

\begin{tabular}{lll}
\hline $\begin{array}{l}\text { Interviewpartnerin/ } \\
\text { Interviewpartner }\end{array}$ & $\begin{array}{l}\text { Anzahl der Airbnb- } \\
\text { Angebote }\end{array}$ & $\begin{array}{l}\text { Kategorie } \\
\text { (vgl. Tabelle 1) }\end{array}$ \\
\hline A 1 & 21 & 4 \\
A 2 & 21 & 4 \\
A 3 & $6-11^{*}$ & 4 \\
A 4 & $5-7^{*}$ & 4 \\
A 5 & 5 & 4 \\
A 6 & 4 & 3 \\
A 7 & 4 & 3 \\
A 8 & 1 & 3 \\
A 9 & 1 & 2 \\
A 10 & 2 & 2 \\
A 11 & 1 & 1 \\
A 12 & 1 & 1 \\
A 13 & 1 & 1 \\
\hline
\end{tabular}

* = Anzahl der Airbnb-Angebote hat sich in den letzten 12 Monaten verändert

Anbietern) repräsentiert die von uns getroffene Auswahl einen signifikanten Teil der Airbnb-Struktur in Salzburg.

Die Themen der leitfadengestützten Interviews waren Motivation, Management des Airbnb-Angebots, Besitzverhältnisse, Nachbarschaft, die öffentliche Debatte zu Airbnb, soziodemographisches Profil der Anbieter. Die Auswertung der Interviews erfolge mit der Datenanalyse-Software MAXQDA. ${ }^{17}$

17 Während der Transkription wurden Memos und anschließend Kodes zu den Themengebieten erstellt. Um die übergeordneten Forschungsfragen zu beantworten, erstellten wir auf der Basis der Kodes einen Kategorienbaum, der als Auswertungsschablone oder 


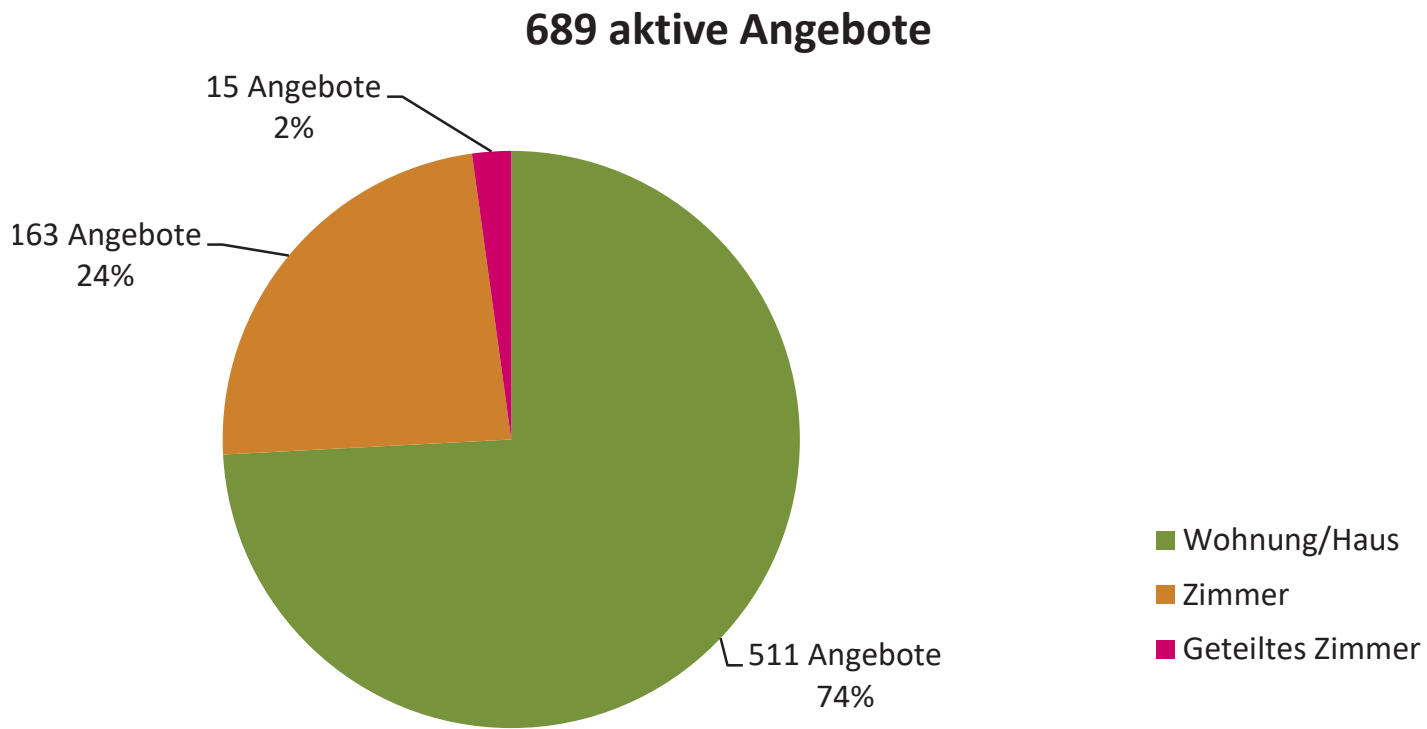

Abbildung 2: Überblick zu den aktiven Angeboten für Kurzzeitvermietungen auf Airbnb nach Angebotstyp in der Stadt Salzburg

\section{Ausgewählte Ergebnisse}

\subsection{Angebotsstruktur und räumliche Verteilung der Airbnb-Angebote in Salzburg}

Die von Airbnb beworbene Idee des geteilten Schlafzimmers oder Wohnraums spielt in Salzburg eine untergeordnete Rolle. Knapp 75 \% der angebotenen 689 Unterkünfte sind komplette Wohnungen oder Häuser. Rund ein Viertel der Angebote (24 \%) sind komplette Zimmer. Das "geteilte Zimmer", bei dem die Mieter sich sowohl Schlafraum als auch den restlichen Wohnraum mit den Vermietern teilen, macht hingegen gerade einmal $2 \%$ (= 15) aus, (vgl. Abbildung 2).

Die räumliche Verteilung von Airbnb-Angeboten in der Stadt Salzburg konzentriert sich auf die touristisch stark frequentierten Innenstadtteile (vgl. Abbildung 1). Diese Verteilung ist analog zu den angebotenen Hotels und Ferienwohnungen auf booking.com. Konkret zeigt die räumliche Analyse, dass es zwei ,Zentren' von Airbnb in Salzburg gibt: innerstädtische Stadtteile und die Bahnhofsgegend. Die hochpreisigen Angebote finden sich in den innerstädtischen Stadtteilen, während es in der Bahnhofsgegend zwar mehr Angebote gibt, diese aber weniger Einnahmen generieren.

Raster bei MAXQDA diente. Im Verlauf der Analyse wurden die Codes und Kategorien sukzessive verfeinert und ergänzt.
Die Verteilung der Angebote nach Größe in Abbildung 3 zeigt, dass drei von vier Anbietern ein Angebot auf Airbnb haben (vgl. innerer Ring in Abbildung 3, hellblau). Rund $19 \%$ der Anbieter haben zwei bis fünf Angebote. $5 \%$ haben mehr als fünf Angebote. Der überwiegende Teil der Anbieter findet sich also im Segment mit nur einem Angebot: Ein Indiz, dass die Hobby-Vermietung - die Vermietung des eigenen Zuhauses - von Relevanz ist.

Betrachtet man jedoch den Airbnb-Markt insgesamt (Angebotsstruktur), das heißt die Anzahl der verwalteten Angebote nach Anbietergröße (vgl. Abbildung 3), zeigt sich ein anderes Bild (vgl. Abbildung 3, äußerer Ring). Obwohl Anbieter mit einem Angebot, wie oben beschrieben, $76 \%$ aller Anbieter ausmachen, stellen diese nicht die Mehrheit der Angebote. Konkret sind es nur rund $45 \%$ aller Angebote. Etwas mehr als ein Viertel (27\%) kommt von Anbietern mit zwei bis fünf Angeboten. Und sogar $28 \%$ entfallen auf Anbieter mit mehr als fünf Angeboten („Top-Anbieter"). Wenn man grob annimmt, dass typische Hobby- Vermieter ein einziges Angebot haben - den eigenen Wohnraum -, kann man demnach rund $45 \%$ der Angebote in der Stadt zu Hobby-Vermietungen zählen und die verbleibenden $55 \%$ zu gewerblichen, kommerziellen Vermietungen. Gewerbliches Vermieten erscheint in diesem Sinne der bestimmende Teil des Airbnb-Marktes in Salzburg zu sein, während in einer vergleichbaren Untersuchung in Wien Hobby-Vermietungen die Mehrzahl der Angebote stellen (vgl. Seidl/ Plank/Kadi 2017). 


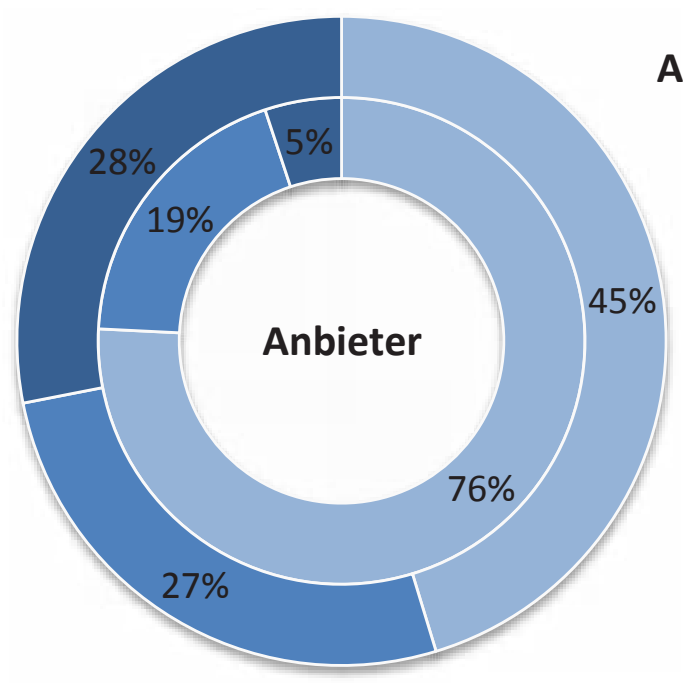

1 Angebot

2-5 Angebote

5+ Angebote

Abbildung 3: Airbnb-Angebote nach Anbietergröße

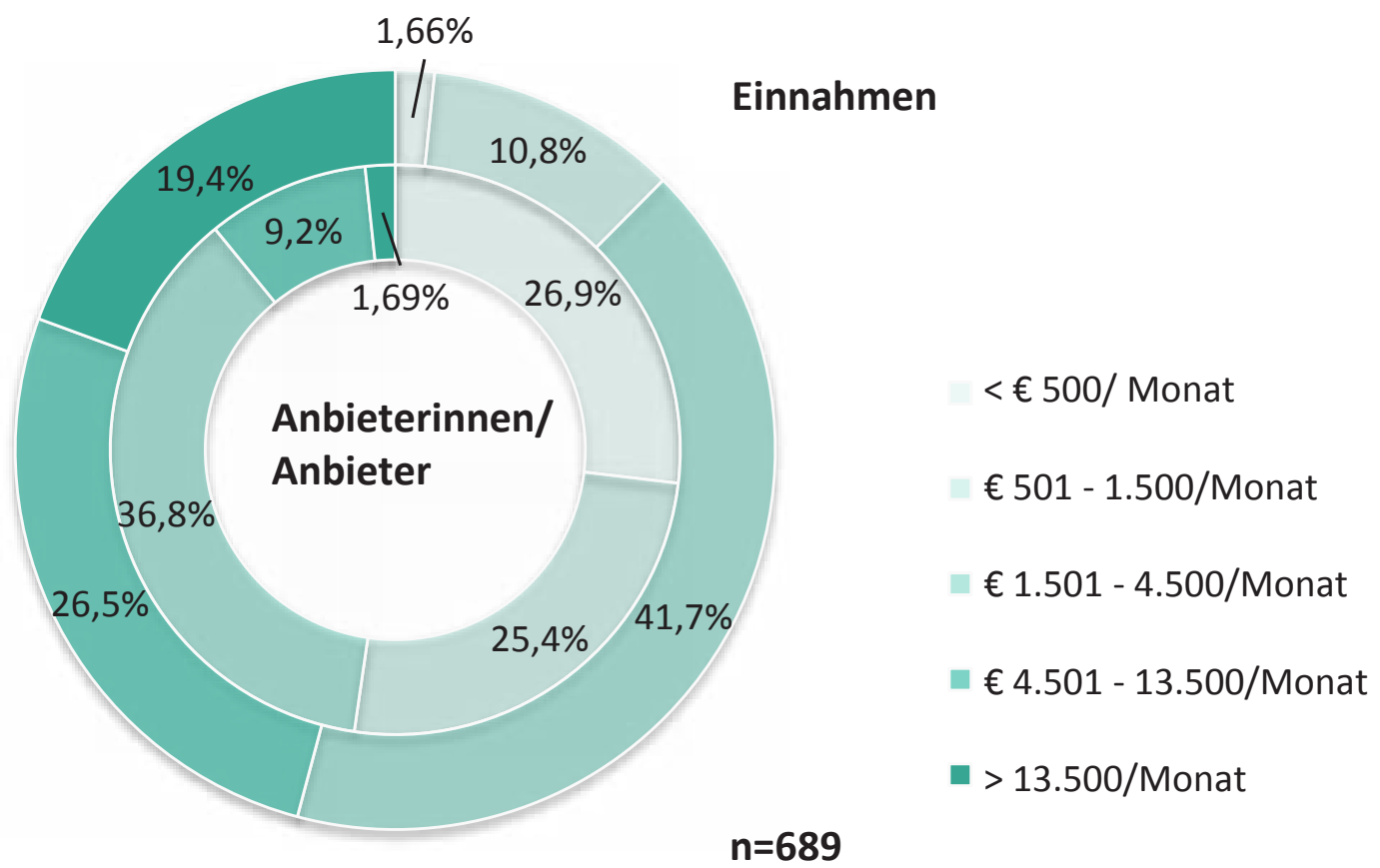

Abbildung 4: Verteilung der Airbnb-Einnahmen auf die Anbieter

Betrachtet man die Verteilung der Einnahmen nach Anbietern, lässt sich eine Konzentration der Einnahmen auf die einnahmestarken Anbieter konstatieren (vgl. innerer Ring in Abbildung 4). Drei von vier Anbietern generieren mehr als 500 Euro pro Monat mittels Airbnb. Nimmt man Einnahmen von bis zu 500 Euro als Nebenverdienst für Hobby-Vermietungen an, so machen diese (nur) gut ein Viertel $(27 \%)$ aller Anbieter aus. Fast die Hälfte kommt auf mindestens 1.500 Euro im Monat.
Noch deutlicher wird die skizzierte Konzentration der Einnahmen, wenn man die Verteilung nach Einnahmegruppen zu Rate zieht (vgl. äußerer Ring in Abbildung 4). Hier zeigt sich eine klare Konzentration der Einnahmen hinsichtlich der einnahmestärksten Anbieter. Fast $88 \%$ der Gesamteinnahmen gehen an Anbieter mit Einnahmen über 1.500 Euro pro Monat. Knapp $46 \%$ der Gesamteinnahmen entfallen auf Vermieter, die mehr als 4.500 Euro verdienen und sogar knapp $20 \%$ der 
Gesamteinnahmen gehen an Anbieter, die mehr als 13.500 Euro im Monat generieren.

Wie auch in anderen Studien deutet bei einigen dieser einnahmestarken Anbietern, die wir als TopAnbieter bezeichnen (mehr als fünf Angebote), bereits der Anbietername bzw. die Beschreibung auf eine gewerbliche Orientierung hin (vgl. Seidl/Plank/Kadi 2017). Für einige findet sich auch eine Nähe zur Hotelbzw. Immobilienbranche (vgl. Kapitel 5.3). Die TopAnbieter in der Stadt Salzburg machen zwar nur rund $5 \%$ der Anbieter aus (vgl. Abbildung 3), verwalten aber mehr als $28 \%$ (absolut 194) aller Angebote. Im Überblick zeigt sich, dass die Spannweite der verwalteten Wohnungsportfolios hier von fünf bis neun Angeboten bei 14 Top-Anbietern bis hin zu 15 bis 21 Angeboten bei den fünf Top-Anbietern an der Spitze reicht. Deren monatliche Einnahmen belaufen sich auf bis zu 44.000 Euro. Klar ist, dass bei Top-Anbietern mit diesem Angebotsvolumen professionelle Strukturen zur Immobilienverwaltung im Hintergrund stehen müssen, um diese - wie von Airbnb forciert - kundenorientiert zu betreiben.

\subsection{Auswirkungen auf den Salzburger Wohnungsmarkt}

Um den Effekt, den Kurzzeitvermietungen für den Wohnungsmarkt haben, genauer zu bestimmen, zielt unsere Analyse auf langfristig vermietete und dem Wohnungsmarkt entzogene Wohnungen. Wir konzentrieren uns daher in der Folge ausschließlich auf den Unterkunftstyp "Wohnung/Haus" (75 \% des gesamten Angebots in der Stadt Salzburg (vgl. Abbildung 2). Angebote aus den Unterkunftstypen "Zimmer" und "geteiltes Zimmer" wurden nicht mit einbezogen. Wie bereits beschrieben, haben wir Schwellenwerte festgelegt (vgl. Kapitel 4.1.5).

Derzeit werden in der Stadt Salzburg - unter den skizzierten konservativen Annahmen - mehr als $50 \%$ $(n=259)$ der angebotenen Wohnungen und Häuser durch Airbnb langfristig dem Wohnungsmarkt entzogen. ${ }^{18}$ Damit kann der Verlust von regulärem Wohnraum im Vergleich zum absolut zur Verfügung stehenden Wohnraum insgesamt betrachtet noch als relativ begrenzt eingestuft werden. Der Wohnraumentzug findet allerdings räumlich stark konzentriert statt. Er betrifft besonders innerstädtische Gebiete.

18 Als konservativ bezeichnen wir unsere Schätzung, da sie neben den weit gefassten Schwellenwerten zudem andere Plattformen für Kurzzeitvermietungen nicht berücksichtigt (z. B. booking.com, auf der einige Interviewpartner ebenfalls vermieten).
Dieser Wert von $50 \%$ der inserierten Wohnungen und Häuser, die dem Wohnungsmarkt durch Airbnb dauerhaft entzogen werden, wird noch aussagekräftiger, vergleicht man inn mit anderen Städten. In der Wiener Studie, die mit der gleichen Methodik gearbeitet hat, liegt dieser Wert bei $38 \%$ (Seidl/Plank/Kadi 2017). Salzburg weist zudem kleinräumig - bezogen auf alle Wohnungen im Zählbezirk - deutlich höhere Anteile von dem Wohnungsmarkt entzogene Wohnungen auf als Wien. In Salzburg betrifft dies insbesondere die dunkelrot markierten Zählbezirke, wo $3 \%$ bzw. 3,7 \% aller Wohnungen durch Airbnb dem Wohnungsmarkt dauerhaft entzogen werden, womit ähnliche Werte wie in der New Yorker Untersuchung von Wachsmuth und Weisler (2018: 1163) erreicht werden (vgl. Abbildung 5).

Insbesondere der Vergleich von potenziellen AirbnbEinnahmen mit den Einnahmen aus regulärer Vermietung macht deutlich, dass durch Kurzzeitvermietung ein erheblicher ökonomischer Druck entsteht, der die Wahrscheinlichkeit erhöht, dass Vermieter sich für eine Airbnb-Vermietung und gegen eine reguläre Vermietung am Wohnungsmarkt entscheiden. Abbildung 6 zeigt, dass grundsätzlich in allen Salzburger Stadtteilen mit Kurzzeitvermietungen mehr erwirtschaftet werden kann als mit regulärer Vermietung. Es besteht also ein stadtweites Einnahmepotenzial, wobei die potenziellen Mehreinnahmen über die Zählbezirke variieren. ${ }^{19}$ Die erwarteten Einnahmen mittels Airbnb übertreffen die regulären Mieteinnahmen teilweise um mehr als 300 \% (z. B. 2.950 Euro pro Monat) in den lukrativen Altstadtgebieten.

In der kritischen Stadtforschung wird diese Differenz zwischen realen und zu erwartenden Einnahmen als rent gap (Ertragslücke) beschrieben, die im Zeitverlauf größer wird, wodurch der ökonomische Anreiz für die Vermieter zu investieren in der Folge steigt, was aber auch zwangsläufig die Wohnpreise steigen lässt und zur Verdrängung von Langzeit-Bewohnern führt (Slater 2017: 120). Im Fall von Airbnb in Salzburg zeigt sich die Besonderheit, dass nicht nur in ausgewählten Gegenden, sondern potenziell sogar stadtweit Ertragslücken entstehen. In diesem Zusammenhang ist die bereits erwähnte New Yorker Studie von Wachsmuth und Weisler (2018) sowohl in

19 Einschränkend ist anzumerken, dass es sich bei den ausgewiesenen Airbnb-Einnahmen um Bruttoeinnahmen ohne Abzüge handelt. Abzuziehen sind im Vergleich zu den Einnahmen aus regulärer Vermietung noch Nebenkosten wie Kosten für Wohnungseinrichtung, Heizung, Strom und Warmwasser oder Reparaturen bei Schäden, die nicht durch den normalen Gebrauch entstanden sind. Damit kommt es bei einem unmittelbaren Vergleich zu einer (leichten) Überschätzung der hier dargestellten Mehreinnahmen. 


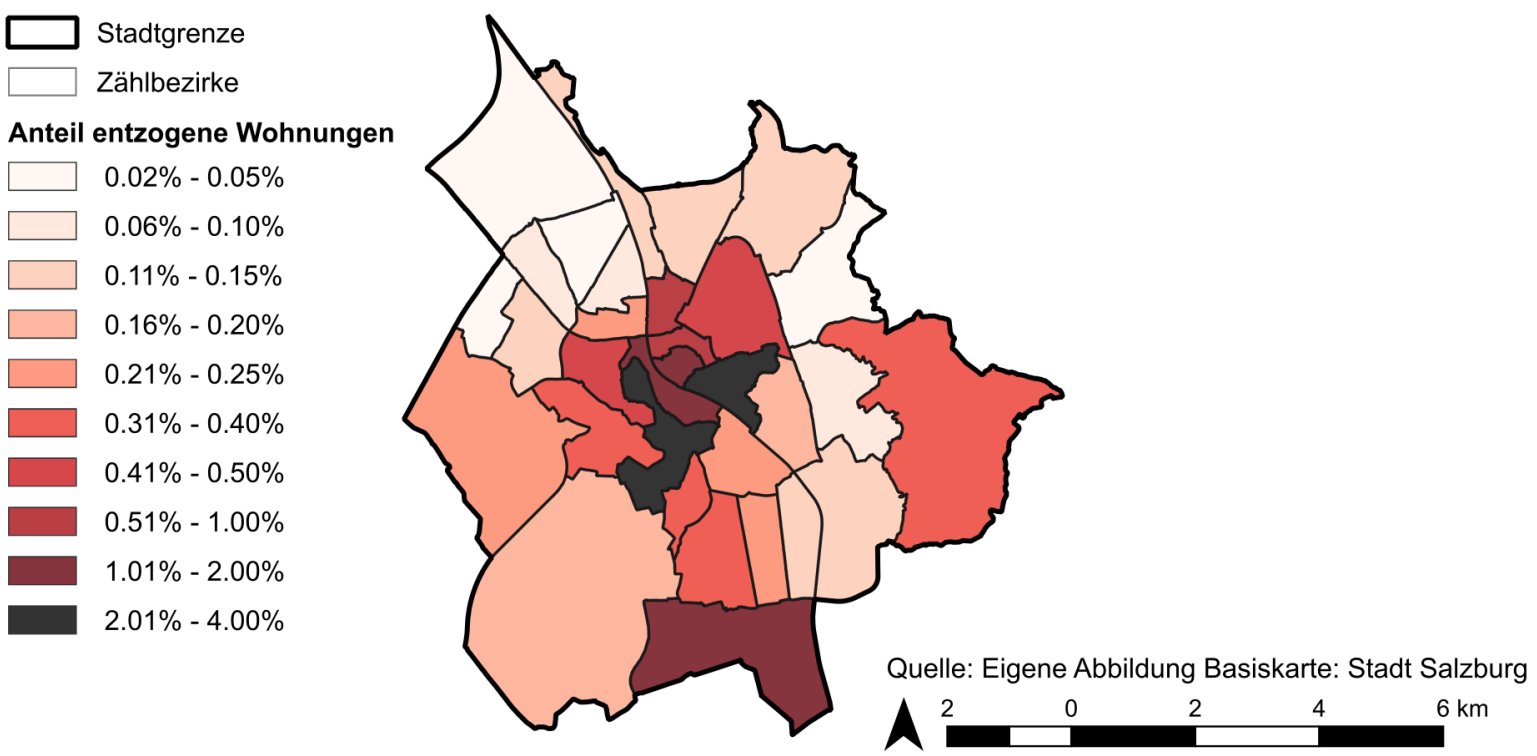

Abbildung 5: Dauerhaft dem Wohnungsmarkt entzogene Wohnungen in Salzburg: Anteil aller Wohnungen im Zählbezirk

konzeptioneller als auch empirischer Hinsicht wegweisend, da sie explizit darauf verweist, dass mittels Airbnb eine neue Form von rent gap entsteht, der nicht mehr eine vorherige Entwertung von Stadtvierteln vorausgeht. Die Ertragslücke ergibt sich nun auch in Stadtgebieten, die sowohl in preislicher als auch soziodemographischer Hinsicht als stabil bezeichnet werden können, da innerhalb sehr kurzer Zeit und mittels geringem finanziellem Aufwand Profitsteigerungen („Ertragslückenschließungen") möglich sind (Wachsmuth/Weisler 2018: 1152). Ob dieses Szenario auch mittels qualitativer Forschung belegt werden kann, werden wir im folgenden Kapitel anhand der Ergebnisse der qualitativen Interviews mit Salzburger Airbnb-Anbietern erörtern.

\subsection{Profile und Motive der Airbnb- Anbieterinnen und Anbieter}

\subsubsection{Mehreinnahmen als ökonomisches Hauptmotiv}

Die Ergebnisse der qualitativen Interviews zeigen, dass die erwarteten und realisierten finanziellen Mehreinnahmen durch Airbnb für fast alle Anbieter aus allen Kategorien das Hauptmotiv darstellen. Sie sind sowohl Ausgangspunkt, warum sie sich für die Airbnb-Vermietung entscheiden, als auch ein Motiv, warum Kurzzeitvermietungen verstetigt oder sogar ausgeweitet werden. Dies gilt sowohl für die kommerziellen Anbieter der Kategorie
3 und 4 als auch für die, die ,nebenberuflich' die eigene Wohnung anbieten bzw. ein Zimmer teilen.

Bei den kommerziellen Anbietern der Kategorien 3 und 4 dominiert die Sichtweise, dass sich mit Kurzzeitvermietungen ein neuer Markt, ein neues lukratives Geschäftsfeld aufgetan hat. Grundsätzlich sind hierfür keine großen Investitionen notwendig, wie einige Interviewte hervorheben. Unabhängig davon haben die kommerziellen Anbieter drei Dinge gemein: Sie verfügen über (liquides) Kapital aufgrund anderer unternehmerischer Tätigkeiten (Hotel, Immobilienwirtschaft, Gastronomie, langjährige Vermietung), besitzen (Wohn-)Eigentum und verfügen über professionelle sowie soziale Kontakte. Häufig ist es eine Kombination dieser drei Eigenschaften, die bei den kommerziellen Anbietern anzutreffen ist. In der Vermietungspraxis findet sich vor allem bei den Top-Anbietern auch ein Angebotsmix aus Eigentumswohnungen plus speziell für die Kurzzeitvermietung angemietete Wohnungen, da Airbnb als wachsendes Marktsegment gesehen und erfahren wird.

„Ja, wir haben eigentlich bei der ersten Wohnung gesehen, dass es ein sehr gutes Geschäft ist oder dass es sehr gut angenommen wird [...] und wir kamen dazu eigentlich, weil mein Mann hat immer schon eigentlich Immobilieninvestments gemacht. [Er] hat auch Sachen gekauft und saniert und [dann] wieder verkauft. Da haben wir gesagt, ja dann machen wir das, wir suchen uns ein Haus, zwei Freunde von uns haben da auch mit investiert und das müss ma sanieren und dann sind sich wirklich fünf Wohnungen ausgegangen, auszubauen“ (A1). 


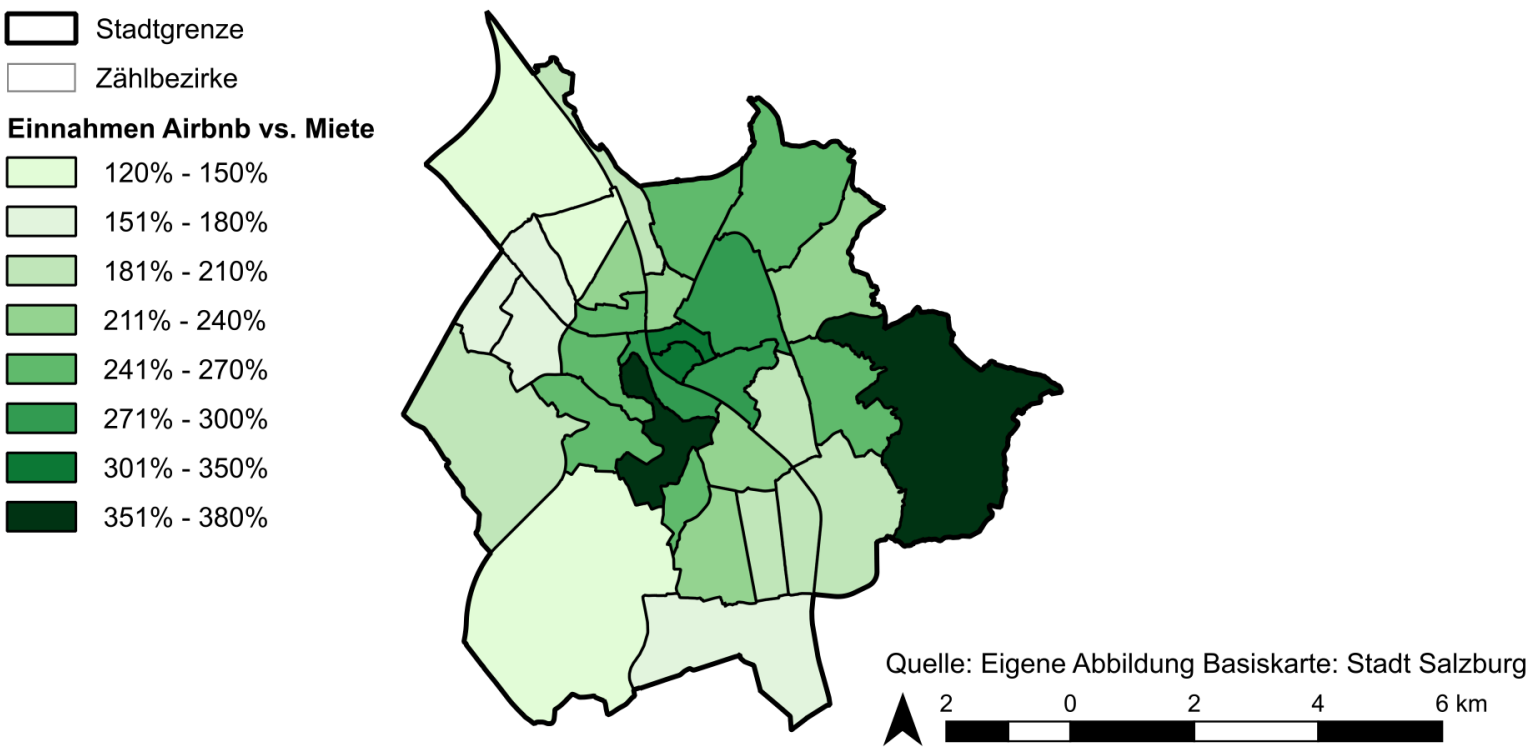

Abbildung 6: Potenzielle Airbnb-Einnahmen und Mieteinnahmen im Vergleich

„Wenn sie das Vierfache verdienen an dem, was sie mit einem Langzeitmieter verdienen, dann ist innen das scheißegal, ob die [Wohnung] sechs Monate leer steht. Völlig belanglos" (A5).

Die zwei Zitate belegen die ökonomische Attraktivität von Kurzzeitvermietungen aus Sicht der Anbieter. Gleichzeitig verdeutlichen sie zwei weitere Aspekte. Einerseits wird die Kurzzeitvermietung via Airbnb, wie bereits erwähnt, als Ausweitung bisheriger Geschäftsfelder bzw. als Investment gesehen, das auch kurzfristig hohe Gewinne abwirft. Es lässt sich schlicht in sehr kurzer Zeit mehr Geld mit kurzfristiger Vermietung verdienen, wodurch eine aktive Verdrängung von ,klassischen' Mietformen bzw. langfristigen Wohnformen geschieht. Zweitens führt dies zu einer Vertiefung von neuen Verwertungspraktiken des Wohnens, die sich sehr dynamisch entwickeln und auch aktiv forciert werden. Teilweise kann eine Form von ,Goldgräberstimmung' ausgemacht werden, was auch die weniger professionellen und kommerziellen Anbieter betrifft.

„[Es] ist einfach so, wenn ich sage, ich will 3.000 Euro haben im Monat und bin damit zufrieden, wenn ich die kriege, wenn man aber 15.000 Euro bekommen hat, weil man das über diese Airbnb-Schiene so kriegt, dann ist man mit dem Anderen nicht mehr zufrieden" (A4).

„Wir haben drei Wohnungen für Einheimische und fünf Wohnungen machen wir über Airbnb schlicht und ergreifend, weil es einfach wirtschaftlich interessant ist. Absolut, nur lieber wäre mir, wenn wir acht Wohnungen [auf Airbnb] hätten, weil das wäre wirtschaftlich noch interessanter, weil ich bin im Kapitalismus aufgewachsen“ (A5).

„Also das sind jetzt keine Wohnungen, die man dem Wohnungsmarkt entziehen kann, weil sie einfach so klein sind. Oder auch von uns aus nicht als Dauerwohnung vermietet werden sollen. Wir würden diese Wohnungen generell maximal drei Monate vermieten und wollen also hier keine Dauermieter haben, die dann ihr soziales Leben aufbauen" (A6).

In den wenigen qualitativen Studien zu Kurzzeitvermietung, die bisher im internationalen Kontext (Berlin, Lissabon, Reykjavik) vorliegen, ist der angespannte Wohnungsmarkt bzw. die prekäre ökonomische Situation der Anbieter stets ein Motiv, warum Wohnungen über Airbnb angeboten werden (Cócola Gant 2016; Mermet 2017; Stors/Kagermeier 2017: 212). Dieser Aspekt spielt bei den Befragten unserer Untersuchung trotz des angespannten Wohnungsmarktes und der hohen Miet- und Kaufpreise in Salzburg interessanterweise keine Rolle. Zwar stellt Airbnb auch für die weniger professionellen und kommerziellen Anbieter der Kategorie 1 einen wichtigen Nebenverdienst dar, allerdings bietet niemand von den Befragten die eigene Wohnung bzw. das eigene Zimmer aufgrund zu geringen Einkommens oder aufgrund prekärer Arbeits- oder Lebensverhältnisse an. Dies widerspricht somit einer von Airbnb selbst verwendeten Argumentation, dass mittels Airbnb vor allem einkommensschwache Haushalte in Städten mit hohen Wohnungspreisen ökonomisch gestärkt werden (Daum 2017: 145). 


\subsubsection{Die Idee der „Sharing Economy“ aus Sicht der Airbnb-Anbieter}

Ein zentraler Bestandteil der Unternehmensphilosophie bzw. Marketingstrategie von Airbnb und anderen Kurzzeitvermietungsplattformen ist die Idee des Teilens von Wohnraum im Sinne einer Sharing Economy, wobei häufig übersehen wird, dass in dieser Idee der Warencharakter der Wohnung bereits immanent vorhanden ist. Der Fokus in der öffentlichen Diskussion liegt jedoch häufig auf dem Teilen und der damit verbundenen Idee eines (vermeintlich) ressourcenschonenden, nachhaltigen Umgangs mit dem Gebrauchsgut Wohnen. Im Rahmen unserer Untersuchung wurden die Anbieter explizit zu diesem Aspekt befragt. Für die kommerziellen Anbieter sind Teilen und Ressourcenschonung, wie die folgenden Zitate stellvertretend zeigen, von keinerlei Bedeutung.

„Ja, also ich glaube zu Vermarktungszwecken ist der Begriff ganz gut, aber die Sharing Economy. Also wenn man wirklich die Anbieter und die Wohnungen kennt, die auf Airbnb sind, dann hat's den Gedanken eigentlich schon komplett verloren. Ich sehe es einfach hauptsächlich als Vertriebskanal, aber weniger als soziale Plattform mit dem Sinn der Sharing Economy" (A3).

„Nein, also das [Sharing Economy] würde ich ja absolut überhaupt nicht wollen. [...] Das geht also so gut, weil hier alles abgetrennt ist. Jede Wohnung hat ihre eigene Eingangstür, ihr eigenes Bad und die eigene Kochnische. Nein, ich würde sagen, das ist halt ein guter Markt und drum biete ich die da an" (A6).

Wie sieht es bei den weniger kommerziellen Anbietern bzw. bei den Homesharern aus? Auch hier spielt der Aspekt des Teilens (mit einer Ausnahme) keine Rolle. Mehrfach wurde der ,kulturelle Austausch" positiv hervorgehoben. Das heißt, die Herkunft der Gäste, das Kennenlernen und der Austausch mit Menschen unterschiedlichen Alters, aus unterschiedlichen Ländern wurden hierbei genannt. Allerdings war dies für niemand von den Befragten der Hauptgrund, warum sie die Wohnungen bzw. Zimmer kurzzeitvermieten. Vielmehr findet sich eine ,pragmatische 'Einstellung, die die Verbesserung der Englischkenntnisse, die angenehmen Gespräche mit den Gästen, den persönlichen Kontakt sehr wohl in einen ökonomischen Kontext stellt.

„Naja, Sharing Economy würde ich das nicht nennen. Ich glaube, es ist sehr wohl eine Möglichkeit Zusatzgeld zu verdienen. Das hat weniger mit ich möchte gern teilen zu tun, glaube ich. Ich glaube, das ist eine rein wirtschaftliche Überlegung" (A11).

\subsubsection{Soziodemographischer Hintergrund der Airbnb-Anbieterinnen und Anbieter}

Bei den Interviewten handelt es sich ausschließlich um Privatpersonen aus Salzburg. 11 von den 13 sind in Salzburg gebürtig, die anderen beiden Interviewten leben aber ebenfalls seit vielen Jahren bzw. Jahrzehnten in der Stadt. Externe Anbieter finden sich in unserer Untersuchung nicht. Diese lokale Anbieterstruktur deckt sich auch mit anderen Untersuchungen zum Salzburger Wohnungsmarkt, die auf die Dominanz lokaler/regionaler Eigentümerstrukturen verweisen (Van-Hametner/ Smigiel/Kautzschmann et al. 2019).

Die Altersstruktur der interviewten Anbieter ist breit gefächert. Sie reicht von 25 bis 65 Jahre. Es gibt keine Dominanz einer bestimmten Altersgruppe. Ein Grund für diese breite Streuung ist die Einfachheit, mit der ein Airbnb-Angebot zu lancieren ist. Technische Hürden gibt es nicht, was von zahlreichen Interviewten unterschiedlichen Alters betont wurde. Ein Airbnb-Angebot ist sprichwörtlich mit zwei Mausklicks getan.

Der berufliche Hintergrund der Interviewten ist vielfältig. Gleichzeitig zeigt sich, dass vor allem die kommerziellen Airbnb-Anbieter überwiegend aus den Bereichen Tourismus/Gastronomie und Immobilienwirtschaft kommen oder freiberuflich tätig sind. Für diese ist Airbnb ein neues Geschäftsmodell oder eine Erweiterung ihrer bisherigen Tätigkeiten auf dem Immobilienmarkt und im Tourismusbereich. In diesem Zusammenhang wird deutlich, dass sich unter den von uns befragten kommerziellen Anbietern keine institutionellen Investoren befinden, die Wohnungen zur Kurzzeitvermietung anbieten. Es handelt sich somit auch bei den Top-Anbietern um lokale/regionale Privatunternehmen. Inwieweit diese lokalen Strukturen der Kurzzeitvermietung in Zukunft aufgebrochen werden bzw. bereits im Umbruch sind, lässt sich momentan noch nicht abschätzen.

Ein weiteres Indiz für die lokale Struktur von Airbnb in Salzburg ist eine gewisse Vernetzung bzw. die gegenseitige Kenntnis der kommerziellen Airbnb-Anbieter untereinander. Insbesondere in Interviews mit Anbietern der Kategorien 3 und 4 wurde direkt auf andere kommerzielle Anbieter verwiesen. Dies reichte von namentlicher Nennung inklusive einiger Details bis hin zur Weitergabe der Kontaktdaten.

„Die Kollegen, die ich kenne, die [haben] in Salzburg so um die sieben Apartments. Ein Anderer hat gerade in Salzburg zwei Häuser gekauft [...] Die haben alle so eine Auslastung von über 80 Prozent" (A4). 
„Naja in Salzburg haben ja mehrere Leute 10 oder 20 Wohnungen. Es ist ja mittlerweile ein Zweig [ ...] denen gehören die Wohnungen ja zum Teil gar nicht, sondern die machen nur die Administration für diese Wohnungen. Das heißt, das ist ein Job und wenn ich heute keinen Bock mehr habe, dann sage ich ok. Wer ist gut im Rennen, dann schaue ich mir das auf Airbnb an, wer vertritt gute Leute, wer vertritt gute Wohnungen, wer hat eine gute Bewertung, dann setzte ich mich mit dem in Verbindung und sag ,hey hast du bock noch fünf Wohnungen zu machen'?" (A5).

\subsubsection{Kommerzialisierung und Professionalisierung}

Wie bereits ausgeführt, haben wir die Airbnb-Anbieter nach ihrem Kommerzialisierungs- bzw. Professionalisierungsgrad unterschieden. Insgesamt zeigen sich Unterschiede zwischen kommerziell und professionell auftretenden Anbietern auf der einen Seite und den Homesharern auf der anderen Seite (vgl. Abbildung 2). Für die Homesharer gilt, dass sie unter anderem weniger stark vernetzt sind und einen intensiveren Kontakt zu den Airbnb-Gästen pflegen als die kommerziellen Anbieter. Darüber hinaus nutzen Homesharer die Kurzzeitvermietung nicht dauerhaft und vermieten nur zu bestimmten Zeiten. Das heißt, sie stellen ihre Wohnung oder ihr Zimmer auch teilweise offline, während die Kommerziellen allesamt dauerhafte Airbnb-Angebote vorhalten.

Die Anbieter der Kategorien 3 und 4 zeichnen sich durch einen hohen Kommerzialisierungs- und Professionalisierungsgrad aus. Vor allem anhand des Professionalisierungsgrades zeigt sich, dass Kurzzeitvermietungen zu einem eigenen Marktsegment geworden sind, an das andere Dienstleistungen und Intermediäre angegliedert sind. Dies reicht vom speziellen Reinigungsservice, der sich um die (tägliche) Reinigung und Instandhaltung der Airbnb-Angebote kümmert, über Angestellte und/oder Software, die mit der Verwaltung der AirbnbBuchungen betraut sind, spezialisierten Rechtsberatungen bis zu Angestellten, die für die Gäste da sind. Die Anzahl der Angestellten variiert bei den kommerziellen Anbietern zwischen zwei und 14.

Neben einer speziellen Buchungssoftware, dem Ausgliedern von Reinigung und „Kundenkontakt“ zeigt sich der Professionalisierungsgrad auch an der Nutzung anderer Buchungsplattformen. Vor allem booking.com wird von den Anbietern der Kategorie 4 (und teilweise auch Kategorie 3) parallel genutzt. Darüber hinaus betreiben die Top-Anbieter teilweise eigene Websites, die auf verschiedene Zielgruppen ausgerichtet sind.
Diese reichen von Familien, über junge Touristengruppen bis hin zu Geschäftsreisenden, wobei insbesondere temporär in Salzburg und Umgebung Arbeitende (zum Beispiel bei der Firma Red Bull) als Zielpublikum genannt wurden.

Mit einem hohen Professionalisierungsgrad geht auch fast immer ein hoher Kommerzialisierungsgrad einher. Das heißt, eine hohe Professionalisierung ist deswegen vorhanden, um die Airbnb-Angebote effektiv, also gewinnmaximierend, zu nutzen.

„Und für die Organisation braucht man eine entsprechende Software. [...] Also eigentlich muss man rund um die Uhr die Woche auch ansprechbar und da sein. Entsprechend organisiert man sich Leute, Mitarbeiter, die das Ganze abdecken. Dann muss einfach die Qualität der Reinigung der Zimmermädchen [stimmen]. Kleinere Reparaturen müssen sofort erledigt werden" (A1).

"So lernt man dann mit der Zeit mit Rücksprache mit anderen Gastgebern, dass man so Schlüsselboxen einrichten lässt. Der einzige große Aufwand ist natürlich der Administrative, den man einfach hat um die Buchungen zu verwalten, Steuer, Ortstaxe etc. [...] das ist ein Aufwand. Der Rest hält sich in Grenzen. Und der zweite Aufwand ist dieses tägliche Hinfahren, Saubermachen, Bettwäsche [...] und da hab "ich mir dann Damen genommen, die das dann so nebenbei machen. Geringfügig“" (A4).

Professionelle bzw. kommerzielle Strukturen lassen sich auch daran ablesen, wie die Airbnb-Anbieter über ihre rechtliche Situation informiert sind, wie sie in dem aktuellen Rahmen agieren und wie sie sich darüber hinaus positionieren. Insbesondere die kommerziellen Top-Anbieter, die einen Angebotsmix aus ,eigenen Wohnungen und angemieteten Wohnungen auf Airbnb anbieten, sind umfassend über die vielfältigen rechtlichen Bestimmungen informiert. Dies reicht von planungsrechtlichen über mietrechtliche bis zu sonstigen steuerrechtlichen Bedingungen. Hierzu wird teilweise professionelle Hilfe in Anspruch genommen.

„Vor einem Jahr habe ich mich bei einem Anwalt beraten, der mir wieder von Anderen empfohlen worden ist, der extra Häuser kauft für dieses Modell. Und da bin ich hin zum Anwalt und habe gefragt, laufe ich da irgendwo Gefahr, sagt er nein also, bei diesen zwei kleinen Häuschen, die Sie haben, das ist, da wird keiner was sagen können. Ich laufe da jetzt nicht Gefahr, ich habe keine Nachbarn. Es wird da nix passieren. In diesem einen, wo ich selber gewohnt habe, da sind drei Parteien drin, da habe ich mir das aber schriftlich geben lassen von den anderen Mietern, dass sie einverstanden sind damit" (A4). 
Insgesamt kann aus den Interviews geschlussfolgert werden, dass sich die Befragten aktiv mit der rechtlichen Situation auseinandergesetzt haben, bevor die Airbnb-Vermietung in Angriff genommen wurde. Keiner der Befragten wähnt sich in einer rechtlich unklaren Situation. Dies gilt auch für die Anbieter der Kategorien 1 und 2. Beispielsweise betonen die Homesharer, dass sie mietrechtliche Absprachen mit ihren Vermietern bzw. Hausverwaltungen getroffen haben. Eine weitere Gemeinsamkeit betrifft die steuerlichen Verpflichtungen, die häufig im Zentrum der öffentlichen Debatte stehen. Insbesondere die von uns befragten kommerziellen Airbnb-Anbieter gaben alle an, Ortstaxe (Beherbergungssteuer) zu entrichten. Diese Aussagen lassen sich zwar nicht überprüfen, sie scheinen aber glaubhaft, da sie freiwillig bei garantierter Anonymität getätigt wurden. Des Weiteren verringern die steuerlichen Abgaben den Gewinn, der gerade durch die kommerziellen AirbnbKurzzeitvermietungen zu machen ist, nur marginal.

"Ich zahle meine Steuern, was ich bei den Hotelketten Sheraton, Ramada, die zum Teil überhaupt nicht in Österreich versteuern [...]. Ich zahle 1,99 Euro jeden Tag pro Person Kurtaxe. Am Jahresende gebe ich meine Buchhaltung ab [...]. Das heißt, ich bin sauberer, was das betrifft, als manche Hotelketten oder manche Hotelgruppen. Das heißt, das Argument, das wir den Hotels was wegnehmen, ja, dann sollen's besser werden, sollen sie billiger werden" (A5).

"Gewerblich nicht. Wir machen die Steuer über meinen Mann und der hat einen Steuerberater und der macht das alles. Mit Ortstaxe, das mache ich, das mit den Steuern das macht dann alles unser Steuerberater, da wird das alles halt dann offengelegt" (A10).

„Nein, es ist eine Mietwohnung. Und es ist ein kleines Haus, wo vier Wohnungen drin sind. Und ich habe dann eben auch mit meinem Vermieter geredet und der hat gesagt, ja passt und diese Renovierung wollte er nicht zahlen. Alles, was Sie machen, hat er gesagt, ist nicht mein Problem" (A12).

\section{Fazit und Ausblick}

Kurzzeitvermietungen stehen gegenwärtig im Fokus der Öffentlichkeit, unter anderem weil das Thema Wohnen eine der drängenden sozialen Fragen unserer Zeit ist (Schönig 2017). Steigende Wohnkosten betreffen bereits seit geraumer Zeit nicht mehr nur boomende Metropolen und die dort lebenden mittleren und unteren Einkommensgruppen. Generell ist in wachsenden Städten und vor allem auch in Universitätsstädten bereits von einer neuen Wohnungsnot die Rede (Birk/Zander/ Lauffer 2018: 5). Ein zunehmend geäußerter Vorwurf in diesem Zusammenhang ist, dass es durch die neue Vermietungspraxis über die Airbnb-Plattform gerade in angespannten Wohnungsmärkten zur Verknappung des Wohnraumangebots kommt. Dementgegen argumentiert eine aktuelle Studie des Instituts der deutschen Wirtschaft, dem Airbnb Daten zur Auswertung zur Verfügung gestellt hat, dass durch Kurzzeitvermietung kein flächendeckender Entzug von Wohnraum nachweisbar sei (BMWi 2018: $67 \mathrm{ff}$.). Dies ist sicherlich verglichen mit anderen Studien auch nicht falsch. Gleichwohl zeigt gerade unsere Studie, dass der gesamtstädtische Fokus eben nicht die adäquate Analyseebene ist. Kurzzeitvermietungen wirken räumlich ungleich. Es sind vor allem ausgewählte (innerstädtische) Stadtviertel, in denen konzentriert Wohnraum entzogen wird. Dies bedeutet auch, dass sozialräumliche Ungleichheiten hierdurch vermutlich weiter verstärkt werden.

In Zahlen ausgedrückt werden nach unserer (konservativen) Schätzung mehr als $50 \%$ der inserierten Wohnungen und Häuser (die $75 \%$ aller Airbnb-Angebote ausmachen) dem Wohnungsmarkt in Salzburg dauerhaft entzogen. Dies betrifft vor allem die innerstädtischen Stadtviertel, wo bereits unter anderem aufgrund des hohen Touristenaufkommens ein Rückgang an Langzeitbewohnern zu beobachten ist.

Die Auswertung der qualitativen Interviews zeigt zudem, dass die Vermieterinnen und Vermieter eine bewusste Entscheidung für Kurzzeitvermietung und gegen Langzeitvermietung getroffen haben. Insbesondere die kommerziellen Anbieter heben hervor, dass Kurzzeitvermietung deutlich höhere Einnahmen generiert. Zudem ist hierfür kein großer finanzieller, baulicher oder rechtlicher Aufwand bzw. Investitionen notwendig, weshalb von den Interviewten explizit langfristige Vermietungsformen als zunehmend unattraktiv eingeschätzt werden. Dies führt in der Folge zur direkten Verdrängung von Wohnraum, was die Interviewten ebenfalls bestätigen und teilweise sogar angaben, das eigene AirbnbAngebot in Richtung Kurzzeitvermietung in Zukunft noch zu erweitern. Diese ersten empirischen Erkenntnisse gilt es, in Zukunft mit weiteren Forschungsarbeiten zu überprüfen. Dies gilt auch hinsichtlich der Erkenntnis unserer Untersuchung, dass der Airbnb-Markt in der Stadt Salzburg gegenwärtig von lokalen, kommerziellen Anbieterinnen/Anbietern dominiert wird. Zudem konnten wir nicht auf die Zusammenhänge zwischen Tourismuspolitik und Stadtpolitik eingehen, die aufgrund ihrer wider- 
sprüchlichen Ausrichtung jedoch Rahmenbedingungen für Kurzzeitvermietungen schaffen.

In konzeptioneller Hinsicht belegt der Beitrag jedoch ein Novum für die Gentrifizierungsforschung, auf das bereits Wachsmuth und Weisler (2018) für New York sowie Yrigoy (2018) für Mallorca hingewiesen haben. In vielen Studien zu Gentrifizierung zeigte sich bisher, dass sich der rent gap (Ertragslücke) zwischen realisierter Grundrente und potenzieller Grundrente mit der Zeit (langsam) vergrößert und dass hierzu unter anderem baulicher Verfall sowie fehlende Investitionen in Infrastruktur beitragen (Smith 1979). Folglich erhöht sich mit der Zeit der ökonomische Anreiz bei Vermietern bzw. Eigentümern zu investieren. Mit Airbnb kommt nun eine gänzlich neue Dynamik ins Spiel, die zu einem abrupten Anstieg der potenziellen Grundrente führt. Wichtig ist hierbei, dass dieser Anstieg ohne große Investitionen bzw. Desinvestitionen vonstatten geht. Die Gewinnaussichten aus Kurzzeitvermietung übertreffen die langfristigen Mieteinnahmen um ein Vielfaches, wie der vorliegende Beitrag empirisch belegt. Die sozialräumlichen Folgen (unter anderem Verdrängung, Nachbarschaftsverhältnisse), die hiermit verbunden sind, wurden bisher kaum untersucht, sie stellen aber ein wichtiges zukünftiges Forschungsthema dar, dem sich die Autorinnen und Autoren dieses Beitrags in einem aktuellen Forschungsprojekt widmen. Erste Studien zeigen, dass beispielsweise die ,langsame' Verdrängung von Langzeitbewohnern durch eine intensivere und schnellere Verdrängung im Fall von Airbnb und ähnlichen Plattformen abgelöst wird (Cócola Gant/Gago 2019). Cócola Gant (2016: 7) hat dies für Barcelona mit dem Terminus "collective displacement" umschrieben. Hierzu zählt neben der Verdrängung von Langzeitbewohnern auch die Verdrängung von Versorgungsinfrastruktur, die auf deren täglichen Bedarf ausgerichtet ist („commercial displacement"), dem erheblichen Verkehrsaufkommen durch Reisebusse und Touristen auch eine „gefühlte Verdrängung“ („place-based displacement“). Die „gefühlte Verdrängung" muss nicht unmittelbar mit dem direkten Verlust der Wohnung einhergehen, sie kann auch alleine aufgrund des enormen ökonomischen Drucks eine Verdrängung des Quartierzusammenhangs bewirken und somit zu einem "loss of place“ bei den dort Lebenden führen (Cócola Gant 2018: 288).

Dieses „collective displacement" ist vor allem für eine massiv touristifizierte Stadt wie Salzburg mit seinen drei Millionen Übernachtungen und neun Millionen Tagestouristen von Bedeutung. Mit Kurzzeitvermietungen (Airbnb) ist ein neuer ,Markt' entstanden, der zwischen Hotelgewerbe und Wohnungsvermietung angesiedelt ist und den vor allem kommerzielle Anbieter bedienen. Konkret zeigt unsere Untersuchung, dass $90 \%$ der Einnahmen an Anbieterinnen/Anbieter gehen, die monatlich mehr als 1.500 Euro mit Airbnb verdienen. Diese kommerzielle Struktur wird sich - auch nach Ansicht der Interviewten - noch verstärken. Konkret wird eine weitere Professionalisierung der Anbieterinnen und Anbieter erwartet. Diesen Umstand gilt es, bei einem weiteren wichtigen Zukunftsthema, der zukünftigen Regulierung von Airbnb mittels politischer und rechtlicher Maßnahmen, zu bedenken. Kleinanbieter sind und werden (nicht nur in Salzburg) zwar weiterhin vorhanden sein, sie sind aus unserer Sicht aber nicht die relevante Zielgruppe in punkto Regulierung.

Danksagung: Der vorliegende Beitrag basiert auf den Ergebnissen eines vom Österreichischen Städtebund geförderten Forschungsprojektes, das von den Autorinnen und Autoren dieses Beitrages von Juni 2018 bis Januar 2019 durchgeführt wurde. Wir danken dem Österreichischen Städtebund für die finanzielle Förderung.

\section{Literatur}

Birk, U.; Zander, C.; Lauffer, F. (2018): Bezahlbares Wohnen. Einführung. In: Informationen zur Raumentwicklung 4, 4-7.

BMWi - Bundesministerium für Wirtschaft und Energie (Hrsg.) (2018): Sharing Economy im Wirtschaftsraum Deutschland. Analyse des Stellenwerts im Allgemeinen sowie Untersuchung der Handlungsoptionen im Einzelsegment 'Vermittlungsdienste für Privatunterkünfte'. Studie des Instituts der deutschen Wirtschaft Köln in Zusammenarbeit mit DICE Consult. Berlin.

Cócola Gant, A. (2016): Holiday Rentals. The New Gentrification Battlefront. In: Sociological Research Online 21, 3, 1-9. doi: 10.5153/sro. 4071

Cócola Gant, A. (2018): Tourism gentrification. In: Lees, L.; Phillips, M. (Hrsg.): Handbook of Gentrification Studies. Cheltenham, 281-293.

Cócola Gant, A.; Gago, A. (2019): Airbnb, buy-to-let investment and tourism-driven displacement. A case study in Lisbon. In: Environment and Planning A. doi: 10.1177/0308518X19869012

Daum, T. (2017): Das Kapital sind wir. Zur Kritik der digitalen Ökonomie. Hamburg.

Dopsch, H.; Hoffmann, R. (2008): Salzburg. Die Geschichte einer Stadt. Salzburg.

EcoAustria - Institut für Wirtschaftsforschung (2019): Konsumausgaben der auf Airbnb buchenden Gäste: Wertschöpfung und Beschäftigung in Österreich. Wien.

Ferreri, M.; Sanyal, R. (2018): Platform economies and urban planning:Airbnb and regulated deregulation in London. In: Urban Studies 55, 15, 3353-3368. doi: 10.1177/0042098017751982

Frisch, T.; Sommer, C.; Stoltenberg, L.; Stors, N. (Hrsg.) (2019): Tourism and everyday life in the contemporary city. London. 
Hörz, M. (2016): Datenjournalistische Nutzung üblicher und unüblicher urbaner Daten. In: Stadtforschung und Statistik 2, 1, 35-42.

Huber, H.; Fröschl, H.; Veigl, S. (2018): Im Luxus-Penthouse mit Airbnb. In: Salzburger Nachrichten vom 3. November 2018, 2-3.

Kagermeier, A.; Köller, J.; Stors, N. (2015): Share Economy im Tourismus: Zwischen pragmatischen Motiven und der Suche nach authentischen Erlebnissen. In: Zeitschrift für Tourismuswissenschaft 7, 2, 117-146. doi: 10.1515/tw-20150203

Kohrs, C. (2019): Vierstellige Beträge für eine Nacht in einer AirbnbWohnung. In: Süddeutsche Zeitung vom 4. Februar 2019, 38.

Mermet, A.-C. (2017): Airbnb and tourism gentrification: critical insights from the exploratory analysis of the 'Airbnb syndrome' in Reykjavík. In: Gravari-Barbas, M.; Guinand, S. (Hrsg.): Tourism and Gentrification in Contemporary Metropolises. London, 52-74.

Neuhold, T. (2018): Salzburger Zentrum ist eine Stadt ohne Bewohner. In: Der Standard vom 21. Januar 2018, 16.

Novy, J.; Colomb, C. (2017): Urban tourism and its discontents: an introduction. In: Colomb, C.; Novy, J. (Hrsg.): Protest and resistance in the tourist city. London, 1-30.

Putschögl, M.; Zoidl, F. (2018): Airbnb: Segen für Urlauber, Fluch für Einheimische. In: Der Standard vom 30. Juni 2018, 31.

Roelofsen, M. (2018): Exploring the Socio-Spatial Inequalities of Airbnb in Sofia, Bulgaria. In: Erdkunde 72, 4, 313-327. doi: 10.3112/erdkunde.2018.04.04

Roland Berger Consulting (2015): European city tourism 2015. Study - analysis and findings. München.

Roland Berger Consulting (2018): Protecting your city from overtourism. European city tourism study 2018. München.

Rossacher, T. (2018): Steiermark leitet rechtliche Schritte gegen Airbnb ein. In: Kleine Zeitung vom 7. November 2018, 32.

Schönig, B. (2017): Sechs Thesen zur wieder mal „neuen“ Wohnungsfrage - Plädoyer für ein interdisziplinäres Gespräch. In: Schönig, B.; Kadi, J.; Schipper, S. (Hrsg.): Wohnraum für alle?! Perspektiven auf Planung, Politik und Architektur. Bielefeld, 11-27. doi: 10.14361/9783839437292-002

Seidl, R.; Plank, L.; Kadi, J. (2017): Airbnb in Wien: eine Analyse. Interaktiver Forschungsbericht, Stand 19. Oktober 2017. http:// wherebnb.in/wien (14.08.2019).

Skowronnek, A.; Vogel, L.; Parnow J. (2015): Airbnb vs. Berlin. Was sagen die Daten? https://airbnbvsberlin.de (14.08.2019).

Slater, T. (2017): Planetary Rent Gaps. In: Antipode 49, S 1, 114-137. doi: 10.1111/anti.12185

Smith, N. (1979): Toward a theory of gentrification: A back to the city movement by capital, not people. In: Journal of the American Planning Association 45, 4, 538-548. doi: 10.1080/01944367908977002

Stadt Salzburg (2013): Einwohner der Stadt Salzburg mit Hauptwohnsitz nach Zählbezirk, Altersgruppe, Geschlecht und Staatsbürgerschaft. Salzburg.

Stadt Salzburg (2018): Statistisches Jahrbuch der Landeshauptstadt Salzburg 2018. Salzburg. = Salzburg in Zahlen 3/2018.

Stadt Salzburg (2019): Der Tourismus im Jahr 2018. Salzburg. = Beiträge zur Stadtforschung 1/2019.

Stors, N.; Kagermeier, A. (2017): Airbnb-Gastgeber als Akteure im ,New Urban Tourism‘. Beweggründe zur Partizipation aus
Anbieterperspektive. In: Geographische Zeitschrift 105, 3/4, 190-224.

Twickel, C. (2018): Auf Ärger eingerichtet. In: Die Zeit vom 16. August 2018, 3 .

Van-Hametner, A.; Smigiel, C.; Kautzschmann, K.; Zeller, C. (2019): Die Wohnungsfrage abseits der Metropolen: Wohnen in Salzburg zwischen touristischer Nachfrage und Finanzanlagen. In: Geographica Helvetica 74, 2, 235-248. doi: 10.5194/gh-74-235-2019

Wachsmuth, D.; Weisler, A. (2018): Airbnb and the rent gap. Gentrification through the sharing economy. In: Environment and Planning A 50, 6, 1147-1170. doi: 10.1177/0308518X18778038

Yrigoy, I. (2018): Rent gap reloaded. Airbnb and the shift from residential to touristic rental housing in the Palma Old Quarter in Mallorca, Spain. In: Urban Studies. doi: 10.1177/0042098018803261

Zeller, C.; Van-Hametner, A.; Smigiel, C.; Kautzschmann, K. (2018): Wohnen in Österreich: von der sozialen Infrastruktur zur Finanzanlage. In: PROKLA. Zeitschrift für kritische Sozialwissenschaft 193, 48, 4, 597-616. doi: 10.32387/prokla. v48i193.1148 